\title{
Novel Algorithms CIPFP for Mining Frequent Patterns using Counting Inference from Probabilistic Databases and Future Possibilities
}

\author{
Niket Bhargava \\ Department of Computer Science and Engineering \\ Mewar University \\ Chittaurgarh, India
}

\author{
Manoj Shukla, $\mathrm{PhD}$ \\ Professor, Department of E\&C \\ BCE, Mandideep \\ Bhopal, India
}

\begin{abstract}
We consider the problem of discovering frequent item sets and association rules between items in a large database of transactional databases acquired under uncertainty. A probabilistic database considered here is one in which with each transaction associated is a probability, represents the confidence that the transaction will occur with given associated certainty. In this paper, we address the problem of the efficiency of the main phase of most data mining applications: The frequent pattern extraction. This problem is mainly related to the number of operations required for counting pattern supports in the database and we propose a new method, called counting inference probabilistic frequent pattern miner in probabilistic databases, this algorithm allows to perform as few support counts as possible. It is optimized to reduce the number of database scan as well as the number of patterns for which explicit support count is required. Using this method, the support of a pattern is determined without accessing the database whenever possible, using the supports of some of its sub-patterns called key patterns. This method was implemented in the CIPFP, counting inference based probabilistic frequent pattern mining algorithm that is an optimization of the simple and efficient Apriori algorithm. The goal is to transform all key patterns into non-key patterns as early as possible as for non-key-patterns database scan is not required at all.
\end{abstract}

\section{General Terms}

Data Mining, Big Data, Data Science, Association Rule Mining,

\section{Keywords}

Probabilistic frequent patterns, probabilistic frequent rule., key-patterns, non-key-patterns,

\section{INTRODUCTION}

Almost all fields of data processing including business data processing is entered in a new era of processing called data science. From simple data processing systems to MIS systems to Data Mining to Big Data systems to Data Science; the goal of data processing and the methods applied are example of systems which are utilizing probability to stabilize system more and more. New area of data processing are coming into picture like predictive analytics and prescriptive analytics. Conclusion is this that, now a days businesses are showing interest in databases that are not only recording exact transactions but at the same time also recording instable, random, inaccurate, incomplete business circumstances into the data and hence with each and every possible transaction are associating a confidence or probability value that indicates strength by which the transaction will took place in real business world. Field of analytics specially predictive and prescriptive analytics playing critical role and are of great use to motivate probabilistic databases. This occurrence of transaction level probability can be evaluated manually as well as automatically or may be in semi-automated mode.

First, Progress in digital bar-coding technology has made it possible for organizations to collect and store massive amounts of sales data, referred to as the basket data. A record in such data typically consists of the transaction date and the items bought in the transaction. Successful organizations view such databases as important pieces of the marketing infrastructure. They are interested in instituting informationdriven marketing processes, managed by database technology, that enable marketers to develop and implement customized marketing programs and strategies [6]. The problem of mining association rules over market basket data was introduced in [4]. An example of such a rule might be that $98 \%$ of customers that purchase tires and auto accessories also get automotive services done. Finding all such rules is valuable for cross-marketing, cross-sell, up-sell, targeted marketing, product bundling and propensity focused attached mailing applications. Other applications include catalog design, addon sales, store layout, and customer segmentation based on buying patterns. The databases involved in these applications are very large. It is imperative, therefore, to have fast algorithms for this task[23].

The following is a formal statement of the problem [4]: Let $I=\left\{i_{1}, i_{2}, \ldots, i_{m}\right\}$ be a set of literals, called items. Let $\mathrm{D}$ be a set of transactions, where each transaction $\mathrm{T}$ is a set of items such that $\mathrm{T} \subseteq \mathrm{I}$. Associated with each transaction is a unique identifier, called its TID. We say that a transaction $\mathrm{T}$ contains $\mathrm{X}$, a set of some items in $\mathrm{I}$, if $X \subseteq T$. An association rule is an implication of the form $X \Rightarrow Y$, where $X \subset I, Y \subset I$, and $X \cap Y=\varnothing$. The rule $\mathrm{X} \Rightarrow \mathrm{Y}$ holds in the transaction set $\mathrm{D}$ with confidence $\mathrm{c}$ if $\mathrm{c} \%$ of transactions in $\mathrm{D}$ that contain $\mathrm{X}$ also contain $\mathrm{Y}$. The rule $X \Rightarrow Y$ has support $s$ in the transaction set $D$ if $\mathrm{s} \%$ of transactions in $\mathrm{D}$ contain $\mathrm{X} \cup \mathrm{Y}$. Our rules are somewhat more general than in [4] in that we allow a consequent to have more than one item[23]. Given a set of transactions $\mathrm{D}$, the problem of mining association rules is to generate all association rules that have support and confidence greater than the user specified minimum support (called minsup) and minimum confidence (called minconf) respectively. Our discussion is neutral with respect to the representation of Database D. For example, D could be a data file, a relational table, or the result of a relational expression[23]. An algorithm for finding all association rules, henceforth referred to as the AIS algorithm, was 
presented in [4]. Another algorithm for this task, called the SETM algorithm, has been proposed in [13]. In this paper, we restudied algorithms, Apriori and Probabilistic_Apriori, that differ fundamentally from these algorithms. The problem of finding frequent item sets and association rules falls within the purview of database mining [3] [12], also called knowledge discovery in databases [21]. Related, but not directly applicable, work includes the induction of classification rules [8] [11] [22], discovery of causal rules [19], learning of logical definitions [18], fitting of functions to data [15], and clustering [9] [10]. The closest work in the machine learning literature is the KID3 algorithm presented in [20]. If used for finding all association rules, this algorithm will make as many passes over the data as the number of combinations of items in the antecedent, which is exponentially large. Related work in the database literature is the work on inferring functional dependencies from data [16]. Functional dependencies are rules requiring strict satisfaction. Consequently, having determined a dependency $X \Rightarrow A$, the algorithms in [16] consider any other dependency of the form $\mathrm{X}+\mathrm{Y} \Rightarrow \mathrm{A}$ redundant and do not generate it. The association rules we consider are probabilistic in nature. The presence of a rule $X \Rightarrow A$ does not necessarily mean that $\mathrm{X}+\mathrm{Y} \Rightarrow \mathrm{A}$ also holds because the latter may not have minimum support. Similarly, the presence of rules $X \Rightarrow Y$ and $Y \Rightarrow Z$ does not necessarily mean that $X \Rightarrow Z$ holds because the latter may not have minimum confidence. There has been work on quantifying the "usefulness" or "interestingness" of a rule [20]. What is useful or interesting is often application-dependent. The need for a human in the loop and providing tools to allow human guidance of the rule discovery process has been articulated, for example, in [7] [14].

Original Apriori works on exact non-probabilistic transactions. But transactions in real world occur with some uncertain probability. Data uncertainty is inherent in applications such as sensor monitoring systems, locationbased services, and biological databases. To record and manage this vast amount of imprecise, uncertain information, probabilistic databases have been recently developed. No commercial implementation of probabilistic databases are available, but many prototype designs are suggested.

In this paper, we study the discovery of frequent patterns and association rules from probabilistic databases under the Possible World Semantics. This is technically challenging, since a probabilistic database can have an exponential number of possible worlds. We evaluated probabilistic apriori algorithms, which discover frequent patterns in bottom-up level by level search manner likewise Apriori.

\subsection{Problem Decomposition and Paper Organization}

In case of non-probabilistic databases the problem of discovering all association rules can be decomposed into two subproblems[4]:

(1). Find all sets of items (itemsets) that have transaction support above minimum support. The support for an itemset is the number of transactions that contain the itemset. Itemsets with minimum support are called large itemsets, and all others small itemsets.

(2). Use the large itemsets to generate the desired rules. Here is a straightforward algorithm for this task. For every large itemset 1 , and all non-empty subsets of 1 . For every such subset $a$, output a rule of the form $a \Rightarrow(1-a)$ if the ratio of support(l) to support(a) is at least minconf. We need to consider all subsets of 1 to generate rules with multiple consequents. Due to lack of space, we do not discuss this subproblem further, but refer the reader to [5] for a fast algorithm.

\section{DISCOVERING LARGE ITEMSETS}

Algorithms for discovering large itemsets make multiple passes over the data. In the first pass, we count the support of individual items and determine which of them are large, i.e. have minimum support. In each subsequent pass, we start with a seed set of itemsets found to be large in the previous pass. We use this seed set for generating new potentially large itemsets, called candidate itemsets, and count the actual support for these candidate itemsets during the pass over the data. At the end of the pass, we determine which of the candidate itemsets are actually large, and they become the seed for the next pass. This process continues until no new large itemsets are found. The Apriori and AprioriTid algorithms differ fundamentally from the AIS[4] and SETM[13] algorithms in terms of which candidate itemsets are counted in a pass and in the way that those candidates are generated. In both the AIS and SETM algorithms, candidate itemsets are generated onthe-fly during the pass as data is being read. Specifically, after reading a transaction, it is determined which of the itemsets found large in the previous pass are present in the transaction. New candidate itemsets are generated by extending these large itemsets with other items in the transaction. However, the disadvantage with the Apriori and AprioriTID algorithms is that they generate the candidate itemsets to be counted in a pass by using only the itemsets found large in the previous pass. The basic intuition is that any subset of a large itemset must be large. Therefore, the candidate itemsets having $\mathrm{k}$ items can be generated by joining large itemsets having k-1 items, and deleting those that contain any subset that is not large. This procedure results in generation of a much smaller number of candidate itemsets. Apriori is explained in next section 2, than in section 3 problem of finding probabilistic frequent itemset is defined and in section 4 problem is solved fully on toy database. Section 5 discusses about novel algorithm CIPFP and CIPFP-Gen with fully solved toy example. Section 6 is for conclusion.

\section{APRIORI ALGORITHM}

In this section we will revisit Apriori algorithm. Notation We assume that items in each transaction are kept sorted in their lexicographic order. It is straightforward to adapt these algorithms to the case where the database DB is kept normalized and each given database record is a 〈TID, item> pair, where TID is the identifier of the corresponding transaction. We call the number of items in an itemset its size, and call an itemset of size $\mathrm{k}$ a k-itemset. Items within an itemset are kept in lexicographic order. We use the notation $\mathrm{c}[1] . \mathrm{c}[2] . \ldots \mathrm{c}[\mathrm{k}]$ to represent a k-itemset $\mathrm{c}$ consisting of items $\mathrm{c}[1], \mathrm{c}[2], \ldots, \mathrm{c}[\mathrm{k}]$, where $\mathrm{c}[1]<\mathrm{c}[2]<\ldots<\mathrm{c}[\mathrm{k}]$. if $\mathrm{c}$ is $\mathrm{XY}$ is an $\mathrm{m}$-itemset, we also call $\mathrm{Y}$ an m-extension of $\mathrm{X}$. Associated with each itemset is a count field to store the support for this itemset. The count field is initialized to zero when the itemset is first created. We summarize in table 1 the notation used in the algorithms. 
Table 1 Notations For Apriori

\begin{tabular}{|c|c|}
\hline k -itemset & An itemset having k items. \\
\hline $\mathrm{L}_{\mathrm{k}}$ & $\begin{array}{c}\text { Set of large k-itemsets } \mathrm{L}_{\mathrm{k}} \\
\text { \#those with minimum support\#. } \\
\end{array}$ \\
$\begin{array}{c}\text { Each member of this set has two fields: } \\
\text { i\# itemset and ii\# support count. }\end{array}$ \\
\hline $\mathrm{C}_{\mathrm{k}}$ & $\begin{array}{c}\text { Set of candidate k-itemsets } \mathrm{C}_{\mathrm{k}} \\
\text { \#potentially large itemsets\#. } \\
\end{array}$ \\
& $\begin{array}{c}\text { Each member of this set has two fields: } \\
\text { i\# itemset and ii\# support count. }\end{array}$ \\
\hline
\end{tabular}

\subsection{Algorithm Apriori}

Figure 1 gives the Apriori algorithm. The first pass of the algorithm simply counts item occurrences to determine the large 1-itemsets. A subsequent pass, say pass k, consists of two phases. First, the large itemsets $\mathrm{L}_{\mathrm{k}-1}$ found in the (k-1)th pass are used to generate the candidate itemsets $\mathrm{C}_{\mathrm{k}}$, using the apriori gen function described in 2.1.1. Next the database is scanned and the support of candidates in $\mathrm{C}_{\mathrm{k}}$ is counted. For fast counting, we need to efficiently determine candidates in $C_{k}$ that are contained in a given transaction $t$. See [5] for a discussion of buffer management.

1) $\mathrm{L}_{1}=\{$ large 1-itemsets $\}$;

2) for $\left(\mathrm{k}=2 ; \mathrm{L}_{\mathrm{k}-1} \neq \Phi ; \mathrm{k}++\right)$ do begin

3) $\mathrm{C}_{\mathrm{k}}=$ apriori-gen $\left(\mathrm{L}_{\mathrm{k}}-1\right)$; // New candidates

4) forall transactions $t \in D$ do begin

5) $\mathrm{C}_{\mathrm{t}}=\operatorname{subset}\left(\mathrm{C}_{\mathrm{k}}, \mathrm{t}\right)$;// Candidates contained in

$\mathrm{t}$

6) forall candidates c $\in \mathrm{C}_{\mathrm{t}}$ do

7) c.count++;

8) end

9) $\mathrm{L}_{\mathrm{k}}=\left\{\mathrm{c} \in \mathrm{C}_{\mathrm{k}} \mid\right.$ c.count $\geq$ minsup $\}$

10) end

11) Answer $=U_{k} L_{k}$;

\section{Figure 1: Algorithm Apriori}

\subsection{The apriori-gen function}

The apriori-gen function takes as argument $\mathrm{L}_{\mathrm{k}-1}$, the set of all large (k-1)-itemsets. It returns a superset of the set of all large k-itemsets. The function works as follows. 1 First, in the join step, we join $\mathrm{L}_{\mathrm{k}-1}$ with $\mathrm{L}_{\mathrm{k}-1}$ : the logic implemented using sql query form is as follows;

(1) insert into $C_{k}$

select p.item[1], p.item[2], ..., p.item[k-1], q.item[k-1]

from $\mathrm{L}_{\mathrm{k}-1} \mathrm{p}, \mathrm{L}_{\mathrm{k}-1} \mathrm{q}$ where p.item $[1]=$ q.item $[1], \ldots$, p.item $[k-2]=q . i t e m[k-$ 2], p.item[k-1] < q.item[k-1];

Next, in the prune step, we delete all itemsets c $\in C_{k}$ itemsets such that some (k-1)-subset of $\mathrm{c}$ is not in $\mathrm{L}_{\mathrm{k}-1}$

(1) forall itemsets c $\in C_{k}$ do

forall (k-1)-subsets s of c do

if ( $\mathrm{c}$ not belongs to $\mathrm{L}[\mathrm{k}-1]$ ) then

delete $\mathrm{c}$ from $\mathrm{Ck}$

\section{PROBABILISTIC FREQUENT AND ASSOCIATION RULE MINING}

In this section we will discuss mining uncertain database data with probabilistic certainty. Data uncertainty is inherent in many applications such as sensor monitoring systems, location-based services, and biological databases. To manage this vast amount of imprecise information, probabilistic databases have been recently developed. In this paper, we study the discovery of frequent patterns and association rules from probabilistic data under the Possible World Semantics. This is technically challenging, since a probabilistic database can have an exponential number of possible worlds. The data managed in many emerging applications is often uncertain. Integration and record linkage tools, for example, associate confidence values to the output tuples according to the quality of matching [34]. In structured information extractors, confidence values are appended to rules for extracting patterns from unstructured data [52]. In habitat monitoring systems, data collected from sensors like temperature and humidity are noisy [34]. The locations of users obtained through RFID and GPS systems are also imprecise [25, 39]. To handle these problems, probabilistic databases have been recently proposed, where uncertainty is treated as a "first-class citizen" $[31,34,44,33,38]$. Due to its simplicity in database design and query semantics, the tupleuncertainty model is commonly used in probabilistic databases. Conceptually, each tuple carries an existential probability attribute, which denotes the confidence that the tuple exists. Figure 2 illustrates this model, which records traffic violation events due to red-light running. The details of each event (e.g., location, and traffic volume) are captured by a red-light camera system, which contains sensors and cameras mounted in road intersections. Each tuple is annotated by a probability 
that a true violation happens. The probability that a violation occurs is determined by sensor measurement errors, as well as the uncertainty caused by automatic information extraction of the photographs taken by the system [53].

\begin{tabular}{|c|c|c|c|c|c|c|}
\hline ID & location & time & speed & traffic & weather & prob. \\
\hline$t 1$ & $\mathrm{x}$ & $8-9 \mathrm{pm}$ & $30-40$ & high & Rain & 0.1 \\
\hline$t 2$ & $\mathrm{x}$ & $7-8 \mathrm{am}$ & $80-90$ & low & null & 1.0 \\
\hline$t 3$ & $\mathrm{x}$ & $8-9 \mathrm{pm}$ & $80-90$ & low & Foggy & 0.5 \\
\hline$t 4$ & $\mathrm{x}$ & $8-9 \mathrm{pm}$ & $30-40$ & high & Rain & 0.2 \\
\hline$t 5$ & $\mathrm{y}$ & $2-3 \mathrm{pm}$ & $50-60$ & low & Sunny & 1.0 \\
\hline
\end{tabular}

Figure 2: A probabilistic database example

To interpret tuple uncertainty, the Possible World Semantics (or PWS in short) is often used [34]. Conceptually, a database is viewed as a set of deterministic instances (called possible worlds), each of which contains a set of zero or more tuples. A possible world for Figure 2 consists of the tuples $\{\mathrm{t} 2, \mathrm{t} 3, \mathrm{t} 5\}$, existing with a probability of $(1-0.1) \times 1.0 \times 0.5 \times(1-0.2) \times$ $1.0=0.036$. Any query evaluation algorithm for probabilistic database has to be correct under PWS. That is, the results produced by the algorithm should be the same as if the query is evaluated on every possible world[34] Although PWS is intuitive and useful, evaluating queries under this notion is costly. This is because a probabilistic database has an exponential number of possible worlds. For example, the table in Figure 1 has $2^{3}=8$ possible worlds. Performing query evaluation or data mining under PWS can thus be technically challenging. In fact, the mining of uncertain or probabilistic data has recently attracted research attention [26]. In [41], efficient clustering algorithms were developed to group uncertain objects that are close to each other. Recently, a Naive Bayes classifier has been developed [49]. The goals of this paper are: (1) propose a definition of frequent patterns and association rules for the tuple uncertainty model; and (2) develop efficient algorithm for mining frequent patterns and association rules.

Figure 3: Sample p-ARs derived from Figure 2

\begin{tabular}{|l|l|}
\hline Association rule & Probability \\
\hline$r 1:\{$ location $=x\} \Rightarrow\{$ time $=8-9 \mathrm{pm}\}$ & 0.15 \\
$r 2: \quad\{$ location $=x\} \quad \Rightarrow \quad$ \{speed $=80-$ & 0.49 \\
90$, traffic $=$ low $\}$
\end{tabular}

The frequent patterns discovered from probabilistic data are also probabilistic, to reflect the confidence placed on the mining results. Figure 3 shows two probabilistic frequent patterns (or p-FP) extracted from the database in Figure 2. A p-FP is a set of attribute values that occur frequently with sufficiently high probabilities. The pmf of the number of tuples is the support count that contains a pattern with specific probability. Under PWS, a database is a set of possible worlds, each of which records a (different) support of the same pattern. Hence, the support of a frequent pattern is a pmf. In figure 1 , if we consider all possible worlds where \{ location $=\mathrm{x}$ \}occurs three times, the pmf of $\{$ location $=$ $\mathrm{x}\}$ with a support of 3 is 0.49 . for the p-FP shown. Figure 3 displays their related probabilistic association rules (or p-ARs). Here, rule $\mathrm{r} 2$ suggests that with a 0.49 probability, 1) red-light violations occur frequently at location $x$ and 2) when this happens, the involved vehicle is likely driving at a high speed amid low traffic. We will later explain more about the semantics of p-FP and $\mathrm{p}$-AR. A simple way of finding p-FPs is to extract frequent pat- terns from every possible world. This is practically infeasible, since the number of possible worlds is exponentially large.

Prior work. [30] studied approximate frequent patterns on noisy data, while [42] examined association rules on fuzzy sets. The notion of a "vague association rule" was developed in [43]. These solutions were not developed on probabilistic data models. For probabilistic databases, $[32,25]$ derived patterns based on their expected support counts. $[54,50]$ found that the use of expected support may render important patterns missing. They discussed the computation of the probability that a pattern is frequent. While [55] handled the mining of single items, our solution can discover patterns with more than one item. The data model used in [50] assumes that for each tuple, each attribute value has a probability of being correct. This is different from the tuple-uncertainty model, which describes the joint probability of attribute values within a tuple. Pmf evaluation method DC algorithm is asymptotically faster than the DP algorithms used in $[54,50]$, and is thus more scalable for large and dense datasets. To our best knowledge, none of the above works considered the important problem of generating association rules on probabilistic databases. This section is organized as follows. Section 3 subsection 1 introduces the notions of p-FPs and p-ARs. Sections 3 subsection 2 present our algorithms for mining p-FPs. Section 3 subsection 3 discusses fully solved example using dataset used in paper introduced Pascal algorithm. The CIPFP algorithm is described in section 5 and example is described in section 6

\subsection{Problem Definition}

We first review frequent patterns and association rules in Sections 3.1.1. Then, we discuss the uncertain data model in Section 3.1.2. We present the problems of mining p-FPs and p-ARs, in Sections 3.1.3 and 3.1.4.

\section{Frequent Patterns and Association Rules}

A transaction is a set of items (e.g., goods bought by a customer in a supermarket). A set of items is also called an itemset or a pattern. Given a transaction database of size $n$ and a pattern $X$, we use $\sup (X)$ to denote the support of $X$, i.e., the number of times that $\mathrm{X}$ appears in the database. A pattern $\mathrm{X}$ is frequent if:

$\sup (X) \geq \operatorname{minsup}(1)$

where minsup $\in \mathrm{N} \cap[1, \mathrm{n}]$ is the support threshold [27].

Given patterns $\mathrm{X}$ and $\mathrm{Y}$ (with $\mathrm{X} \cap \mathrm{Y}=\varnothing$ ), if pattern $\mathrm{XY}$ is frequent, then $X$ is also frequent (called the anti-monotonicity property). Also, $X \Rightarrow Y$ is an association rule if following conditions holds:

$\operatorname{supp}(\mathrm{XY}) \geq \operatorname{minsup}$

$\operatorname{supp}(\mathrm{XY}) / \operatorname{supp}(\mathrm{X}) \geq \operatorname{minconf}$

$\sup (X Y) / \sup (X)$, denoted by $\operatorname{conf}(X \Rightarrow Y)$, is the confidence of $X \Rightarrow Y$, and minconf $\in R \cap(0,1]$ is the confidence threshold. To verify Equation 3, the values of $\sup (\mathrm{XY})$ and $\sup (X)$ have to be found first.

We remark that a transaction database is essentially a relational table with asymmetric binary attribute values. 
For example, the existence of item "apple" in a transaction is equivalent to a binary attribute of a tuple with a value of 1 . This kind of attributes, assumed in this paper, is also considered by some mining algorithms (e.g., [27, 28]). To handle other attribute types (e.g., continuous and categorical), discretization and binarization techniques can be used to convert them to binary attributes [52].

\subsection{The Possible World Semantics}

We assume that each transaction has an existential probability, which specifies the chance that the transaction exists. Figure 4(a) illustrates this database, in which each transaction is a set of items represented by letters. This model has been used to capture uncertainty in many applications, including data streams[33] and geographical services[45].

Now, let $\mathrm{P}(\mathrm{E})$ be the probability that an event $\mathrm{E}$ occurs and PDB be probabilistic database of size n. Also, let Ti (where $\mathrm{i}=1, \ldots, \mathrm{n}$ ) be the ID of each tuple in PDB. Suppose Ti.S is the set of items contained in Ti, and Ti.p is the existential probability of Ti.

Figure 4(a) A probabilistic database

\begin{tabular}{|l|l|l|}
\hline ID & SetOfItems & $\begin{array}{l}\text { Probability/ } \\
\text { confidence }\end{array}$ \\
\hline T 1 & $\{\mathrm{a}, \mathrm{c}, \mathrm{e}, \mathrm{g}, \mathrm{i}\}$ & 0.6 \\
\hline T 2 & $\{\mathrm{a}, \mathrm{c}, \mathrm{f}, \mathrm{h}\}$ & 0.5 \\
\hline T 3 & $\{\mathrm{a}, \mathrm{d}, \mathrm{e}, \mathrm{g}, \mathrm{j}\}$ & 0.7 \\
\hline T 4 & $\{\mathrm{b}, \mathrm{d}, \mathrm{f}, \mathrm{h}, \mathrm{i}\}$ & 1.0 \\
\hline
\end{tabular}

Under PWS, PDB is a set of possible worlds W. Figure 4(b) lists all possible worlds for figure 4(a). Each Wi $\varepsilon$ $\mathrm{W}$ exists with probability $\mathrm{P}(\mathrm{Wi})$. For example $\mathrm{P}(\mathrm{W} 2)=$ T1.p X ( 1 - T2.p) X ( 1 -T3.p) X T4.p or 0.09. The sum of possible world probabilities is one. Also, the number of possible worlds is exponentially large, i.e. $|\mathrm{w}|=\mathrm{O}($ $2^{\mathrm{n}}$ ). Our goal is to discover patterns and rule using these possible worlds.

Figure 4(b) Possible World for PDB in Figure 4(a)

\begin{tabular}{|c|c|c||c|c|c|}
\hline W & Tuples in $W$ & Prob. & W & Tuples in $W$ & Prob. \\
\hline$W 1$ & $T 4$ & 0.06 & $W 5$ & $T 1, T 2, T 4$ & 0.09 \\
\hline$W 2$ & $T 1, T 4$ & 0.09 & $W 6$ & $T 1, T 3, T 4$ & 0.21 \\
\hline$W 3$ & $T 2, T 4$ & 0.06 & $W 7$ & $T 2, T 3, T 4$ & 0.14 \\
\hline$W 4$ & $T 3, T 4$ & 0.14 & $W 8$ & $T 1, T 2, T 3, T 4$ & 0.21 \\
\hline
\end{tabular}

\subsection{Probabilistic Frequent Patterns}

we first explain the concept of support for probabilistic data. Given a pattern $X$, we denote its support in each world $\mathrm{Wi}$ as $\sup _{\mathrm{i}}(\mathrm{X})$ is obtained by counting the number of times $X$ appears in Wi. Since each Wi exists with a probability, the support of $X$ in $\mathrm{PDB}$, i.e. $\sup (\mathrm{X})$, is a random variable. We denote $f_{X}(k)$ that the probability mass function (pmf) of $\sup (\mathrm{X})$ can take. Specifically,
$f_{X}(k)$ is the probability that $\sup (X)=k$, and $f_{X}(k)=0$ for any $\mathrm{k} \notin[0, \mathrm{n}]$. We use an array to store the non-zero values of $f_{X}$, where $f_{X}[k]=P(\operatorname{supp}(X)=k)$. Figure 4(c) depicts the support pmf of $\{a\}$. the probability that $\sup (\{\mathrm{a}\})=1$ is 0.29 .

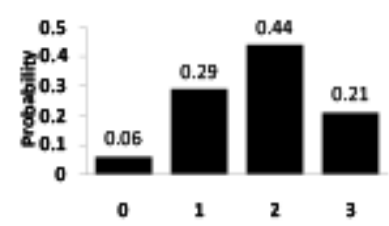

Figure 4(C) Support pmf for 1-itemset $\{$ a $\}$ in PWS

DEFINITION 1. A pattern $X$ is a probabilistic frequent pattern or p-FP in PDB if

$\mathrm{P}(\sup (\mathrm{X}) \geq \operatorname{minsup}) \geq \operatorname{minprob}$

where minprob $\epsilon \mathrm{R} \cap(0,1]$ is the probability threshold.

Problem 1 (p-FP Mining). Given PDB, minsup and minprob, return a set of $\left\{X, f_{X}(k)\right.$, where $X$ is a $p$-FP .As we will discuss, the pmfs obtained with p-FPs are essential to generating probabilistic association rules. There are methods to approximating and compressing pmfs (e.g., see [35]). Here we assume that the pmf is exact, but our solutions can be extended to support these schemes. Next, we present a useful lemma.

Lemma 1 (Anti-monotonicity). If pattern $X$ is a p-FP, then any pattern $\mathrm{X}^{\prime} \subset \mathrm{X}$ is also a p-FP.

The anti-monotonicity property is true for frequent patterns in exact data [27]. Lemma 1 allows us to stop examining a pattern, if any of its sub-pattern is not a p-FP. A p-FP X is said to be maximal if we cannot find another p-FP Y such that $\mathrm{X} \subset \mathrm{Y}$. A maximal $\mathrm{p}$-FP can succinctly represent a set of $\mathrm{p}$ FPs when their supports are not concerned. Since the mining of maximal frequent patterns is an important problem [28] for exact data, we also study maximal p-FPs, together with freeset maximal set make a complete system, we study them too,

Problem 2.1 (Free set p-FP Mining). Given a database PDB, minsup and minprob, return all minimal generators or free sets p-Fps.

Problem 2.2 (Maximal p-FP Mining). Given a database PDB, minsup and minprob, return all maximal p-Fps.

Problem 2.3 (closed p-FP Mining). Given a database PDB, minsup and minprob, return all closed p-FPs.

Probabilistic Association Rules

In a probabilistic database, the support counts of patterns are random variables. Let $P(X \Rightarrow Y)$ be the probability that $X \Rightarrow Y$ is an association rule. By Equations 2 and 3, we have:

$P(X \Rightarrow Y)=P[\sup (X Y)>\operatorname{minsup} \wedge \operatorname{conf}(X \Rightarrow Y) \geq \operatorname{minconf}]$ (5)

Definition 2. $\mathrm{X} \Rightarrow \mathrm{Y}$ is a probabilistic association rule (pAR in short) if

$\mathrm{P}(\mathrm{X} \Rightarrow \mathrm{Y}) \geq$ minprob

The problem of p-AR mining is defined as follows. 
Problem 3 (p-AR Mining). Given minsup, minprob, minconf, and the p-FPs and their support pmfs obtained from Problem 1, derive all $\mathrm{p}$-ARs and their probabilities.

A simple way of solving Problems 1, 2, and 3 is to expand PDB into all possible worlds, compute patterns and rules from each world, and then combine the results. If minsup $=2$, minconf $=0.5$, and minprob $=0.2$, for Figure $4(a),\{a\} \Rightarrow\{c\}$ is an association rule only in worlds W5 and W8 (Figure 4(c)), with $\mathrm{P}(\{\mathrm{a}\} \Rightarrow\{\mathrm{c}\}=\operatorname{Pr}(\mathrm{W} 5)+\operatorname{Pr}(\mathrm{W} 8)=0.09+0.21=0.3$. Since this is larger than $0.2,\{a\} \Rightarrow\{c\}$ is a p-AR. This method is not practical, due to the large number of possible worlds. To tackle Problems 1 and 2, proposed algorithms, namely probabilistic Apriori discussed and solved fully in next section 4.

\section{PROBABILISTIC APRIORI USING PWS FULLY SOLVED CASE}

In this section we display the probabilistic Apriori using PWS fully Solved using toy database used in research paper by Bastides et. al. described algorithm Pascal. To solve Problem 1 , we discussed the probabilistic-Apriori algorithm, which is an adaptation of the Apriori algorithm [27] for probabilistic databases. Specifically, probabilistic-Apriori uses the bottomup framework [27]: using PWS.

Our Probabilistic Algorithm: ProbabilisticApriori

1. PDB Database making,

2. PWS making using PDB,

3. Define candidate $\mathrm{C}_{1}$ 1-itemset equal to Item Collection I.

4. PDB database scan for all $\mathrm{c}$ belongs to $\mathrm{C} 1$ to calculate support c.support. Store them in a list call it FP.

5. PWS database scan for all $\mathrm{c}$ in FP and all c.support where support is equal to "0" to "c.support" to evaluate fc[k] probability pmf support.

6. Determining Probabilistic Frequent Patterns using minsup and minprob. First compare minsup with c.support. If c.support $\geq$ minsup than using pmf for pattern $\mathrm{c}$ in $\mathrm{C} 1$ compare $\mathrm{fc}[\mathrm{k}] \geq$ minprob. Collect all $\mathrm{c}$ in PFP and assign it to $\mathrm{L}_{1}$ all along with valid frequent probable support and probability. Call it $\mathrm{L}_{1}$.

7.1) $\mathrm{L}_{1}=\{$ large 1-itemsets $\}$;

7.2) for $\left(\mathrm{k}=2 ; \mathrm{L}_{\mathrm{k}-1} \neq \Phi ; \mathrm{k}++\right)$ do begin

7.3) $\quad \mathrm{C}_{\mathrm{k}}=$ apriori-gen $\left(\mathrm{L}_{\mathrm{k}}-1\right)$; // New candidates

7.4) forall transactions $\mathrm{t} C \mathrm{PDB}$ do begin

7.5) $\mathrm{C}_{\mathrm{t}}=\operatorname{subset}\left(\mathrm{C}_{\mathrm{k}}, \mathrm{t}\right) ; \quad / /$ Candidates contained

in $\mathrm{t}$

7.6) forall candidates c $\in \mathrm{C}_{\mathrm{t}}$ do

7.7) c.count++;

7.8) end

7.9) $\mathrm{FP}=\left\{\mathrm{c} \in \mathrm{C}_{\mathrm{k}} \mid\right.$ c.count $\geq$ minsup $\}$

7.10) forall $\mathrm{fp} \in \mathrm{FP}$

7.11) from $\mathrm{k}=0$ to fp.support

7.12) $\quad \mathrm{W}=\mathrm{w} \in \mathrm{PWS}$ exactly with size $\mathrm{k}$

times $\mathrm{k}$ number of transactions
Ffp[k].prob++;

7.15) $\quad \mathrm{PFP}=\left\{\mathrm{FP} \in \mathrm{FP}_{\mathrm{k}} \mid \mathrm{Ffp}[\mathrm{k}] \cdot\right.$ prob $\geq$ minprob $\}$

7.15) $\mathrm{L}_{\mathrm{k}}=\mathrm{PFP}$

10) end

11) Answer $=U_{k} L_{k}$;

Figure 5: Probabilistic Apriori Algorithm

\section{The apriori-gen function}

The apriori-gen function takes as argument $\mathrm{L}_{\mathrm{k}-1}$, the set of all large (k-1)-itemsets. It returns a superset of the set of all large $\mathrm{k}$-itemsets. The function works as follows. 1 First, in the join step, we join $\mathrm{L}_{\mathrm{k}-1}$ with $\mathrm{L}_{\mathrm{k}-1}$ : the logic is in the form of sql query form is as follows;

(1) insert into $\mathrm{C}_{\mathrm{k}}$

select p.item[1], p.item[2], ..., p.item[k-1], q.item[k-1]

from $L_{k-1} p, L_{k-1} q$

where p.item $[1]=$ q.item $[1], \ldots$, p.item $[\mathrm{k}-2]=$ q.item[k-2], p.item[k-1] < q.item[k-1];

Next, in the prune step, we delete all itemsets c $\in C_{k}$ itemsets such that some (k-1)-subset of $\mathrm{c}$ is not in $\mathrm{L}_{\mathrm{k}-1}$.

(1) forall itemsets c $\in C_{k}$ do forall (k-1)-subsets s of $\mathrm{c}$ do

$$
\text { if ( } c \text { not belongs to } \mathrm{L}_{\mathrm{k}-1} \text { ) then }
$$

delete $\mathbf{c}$ from $\mathbf{C}_{\mathrm{k}}$;

\subsection{Solved Example Probabilistic Apriori}

This section discusses the solved example of probabilistic Apriori on toy database. To explain fully general version is solved to full length. The database used is same as used is paper which presented Pascal Algorithm extended to include the transaction level existential probability and time stamp for time at which transaction took place. Figure 6 depicts Pascal Transaction Temporal Dataset with Probabilistic Confidence. For our Probabilistic Apriori algorithm only column TID, SetOfItems, and Probabilistic/Confidence are of importance or relevant.

Figure 6. PDB for Pascal Transactional Temporal Dataset with Probabilistic Confidence

\begin{tabular}{|l|l|l|l|l|}
\hline TID & SetOfItems & $\begin{array}{l}\text { TimeSta } \\
\text { mp }\end{array}$ & Key & Probability/Confidence \\
\hline T1 & A, B, C, F & 1 & $?$ & 0.6 \\
\hline T2 & B, C, E, F & 2 & $?$ & 0.5 \\
\hline T3 & $\begin{array}{l}\text { A, B, C, E, }, \\
\text { F }\end{array}$ & 3 & $?$ & 0.7 \\
\hline T4 & B, E, F & 4 & $?$ & 0.4 \\
\hline T5 & $\begin{array}{l}\text { A, B, C, E, }, \\
\text { F }\end{array}$ & 5 & $?$ & 1.0 \\
\hline
\end{tabular}

Using concepts of Possible World Semantics in earlier sections, on database presented in Figure 6 we calculated PWS, which is available in Figure 7. 
Figure 7. PWS for PDB for Pascal Transactional Temporal Dataset with Probabilistic Confidence

\begin{tabular}{|c|c|c|c|}
\hline $\begin{array}{l}\text { Worl } \\
\text { ds }\end{array}$ & $\begin{array}{l}\text { Transactio } \\
\text { nInWorld }\end{array}$ & WorldProbalityCalculation & $\begin{array}{l}\text { WorldProbab } \\
\text { lity }\end{array}$ \\
\hline W1 & T5 & $\begin{array}{l}(1-0.6) *(1-0.5) *(1-0.7) *(1- \\
0.4) *(1.0)\end{array}$ & 0.036 \\
\hline W2 & T1T5 & $\begin{array}{l}(0.6) *(1-0.5)^{*}(1-0.7) *(1- \\
0.4)^{*}(1.0)\end{array}$ & 0.054 \\
\hline W3 & T2T5 & $\begin{array}{l}(1-0.6) *(\quad 0.5 \quad) *(1-0.7) *(1- \\
0.4)^{*}(1.0)\end{array}$ & 0.036 \\
\hline W4 & T3T5 & $\begin{array}{l}(1-0.6) *(1-0.5) *(\quad 0.7 \quad) *(1- \\
0.4)^{*}(1.0)\end{array}$ & 0.084 \\
\hline W5 & T4T5 & $\begin{array}{l}(1-0.6) *(1-0.5) *(1-0.7) *(\quad 0.4 \\
) *(1.0)\end{array}$ & 0.024 \\
\hline W6 & T1T2T5 & $\begin{array}{l}\left(\begin{array}{c}0.6 \\
) *(\end{array} 0.5\right) *(1-0.7) *(1- \\
0.4)^{*}(1.0)\end{array}$ & 0.054 \\
\hline W7 & T1T3T5 & $\begin{array}{l}(0.6) *(1-0.5) *\left(\begin{array}{ll}0.7 & 0 . *(1- \\
0.4) *(1.0)\end{array}\right.\end{array}$ & 0.126 \\
\hline W8 & T1T4T5 & $\begin{array}{l}(0.6) *(1-0.5) *(1-0.7) *(0.4 \\
) *(1.0)\end{array}$ & 0.036 \\
\hline W9 & T2T3T5 & 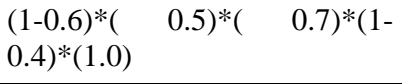 & 0.084 \\
\hline W10 & T2T4T5 & $\begin{array}{l}(1-0.6) *(0.5) *(1-0.7) *(0.4 \\
) *(1.0)\end{array}$ & 0.024 \\
\hline W11 & T3T4T5 & $\begin{array}{l}(1-0.6) *(1-0.5) *(0.7) *(0.4 \\
) *(1.0)\end{array}$ & 0.056 \\
\hline W12 & T1T2T3T5 & $\begin{array}{l}(0.6) *\left(\begin{array}{ll}0.5 \\
(\end{array}\right) *\left(\begin{array}{ll}0.7 & *\end{array}\right) *(1- \\
0.4)^{*}(1.0)\end{array}$ & 0.126 \\
\hline W13 & T1T2T4T5 & $\begin{array}{l}(0.6) *(0.5) *(1-0.7) *(0.4 \\
) *(1.0)\end{array}$ & 0.036 \\
\hline W14 & T1T3T4T5 & $\begin{array}{l}(0.6) *(1-0.5) *(0.7) *(0.4 \\
) *(1.0)\end{array}$ & 0.084 \\
\hline W15 & T2T3T4T5 & $\begin{array}{l}(1-0.6) *(0.5) *(0.7) *(0.4 \\
) *(1.0)\end{array}$ & 0.056 \\
\hline W16 & $\begin{array}{l}\text { TIT2T3T4T } \\
5\end{array}$ & $\begin{array}{l}(0.6) *(0.5) *(0.7) *(0.4 \\
) *(1.0)\end{array}$ & 0.084 \\
\hline
\end{tabular}

The collection of Items for figure 6 PDB contains 6 individual items, let say $\mathrm{I}$ is the set of these items. Hence $\mathrm{I}=\{\mathrm{A}, \mathrm{B}, \mathrm{C}$, $\mathrm{D}, \mathrm{E}, \mathrm{F}\}$, For this example minsup is $2 / 5$ that is $40 \%$. As total number of transactions in database i.e. the size of database $n$ is 5 . So, the threshold for minsup will be given by $(5 * 40)$ $/ 100$, i.e 2 . So, minsup is 2 , let minprob is 0 . We are taking minprob 0 to show that algorithm will behave exactly as apriori behave when no probabilistic transaction is considered. This is equivalent to treating all transactions having certainty or probability of 1 to occur.

All the elements which belongs to collection I will become the candidate pattern, as the individual items themselves are used as patterns we call them 1-itemset. The collection of candidate 1-itemset denoted as $C_{1}$ is as follows: $C_{1}=\{\{A\}$, $\{B\},\{C\},\{D\},\{E\},\{F\}\}$ for each element $c$ belongs to $C_{1}$ we calculate its support in database PDB Figure 6. For all itemset the PDB database scan output is as follows:

\begin{tabular}{|l|l|}
\hline Pattern/1-itemset & Support in PDB \\
\hline$\{A\}$ & 3 \\
\hline$\{B\}$ & 4 \\
\hline$\{C\}$ & 4 \\
\hline$\{D\}$ & 1 \\
\hline$\{E\}$ & 4 \\
\hline$\{F\}$ & 5 \\
\hline
\end{tabular}

Figure 8. $C_{1}$ and its Support Count

In the figure 8 if we compare support of respective patterns we found that pattern $\{D\}$ has a support 1 which is less than the minimum support. $\sup (\{\mathrm{D}\})<\operatorname{minsup}$. So the set of frequent 1-itemset $\mathrm{FP}_{1}$ will contain all 1-itemset from figure 8 but not $\{\mathrm{D}\}$.This set of frequent 1-itemset $\mathrm{FP}_{1}$ is as follows;

\begin{tabular}{|l|l|}
\hline Pattern/1-itemset & Support in PDB \\
\hline$\{A\}$ & 3 \\
\hline$\{B\}$ & 4 \\
\hline$\{C\}$ & 4 \\
\hline$\{E\}$ & 4 \\
\hline$\{F\}$ & 5 \\
\hline
\end{tabular}

Figure 9 FP $_{1}$ set of frequent 1-itemset

Now we use patterns in $\mathrm{FP}_{1}$ one by one to calculate their existential probability. For this we first scan PDB and extract the TIDs in which pattern is present. For combination of length equal to in range for support from 0 to c.support we start scan of PWS rows and sum up the provabilities of Wi in which exactly the number of transaction present, here the number of transaction which occurred in $\mathrm{Wi}$ is determined for every support from 0 to c.support. For example we want to determine existential probability of frequent 1 -itemset $\{\mathrm{A}\}$ to determine probabilistic frequent pattern $\{\mathrm{A}\}$ is or not. First, we scan PDB and found that T1, T3, T5 contains $\{\mathrm{A}\}$. $\{\mathrm{A}\}$.support is 3 . So for transaction combination length of 0 , 1,2 , and 3 , are possible $\mathrm{k}$ th support for $\{\mathrm{A}\}$ in PDB. Let say for example support 1 of pattern $\{A\}$, exactly for the count of 1 , number of transactions that have $\{\mathrm{A}\}$, Wi will form universe, means any Wi that contain exactly one time any of the $\{\mathrm{A}\}$.Ti, i.e., Wi exactly contain either one of $\mathrm{T} 1$, or $\mathrm{T} 2$, or $\mathrm{T} 2$ but not T1T2, T1T3 or T2T3 or T1T2T3 together, all their probabilities will be summed up. For $\{\mathrm{A}\}$.support is 1, PWS is W1W3W5W10 with probability $0.036,0.036,0.024,0.024$ summed up to 0.12 , this 0.12 is existential probability of pattern $\{\mathrm{A}\}$ when its support is 1 . Like wise we calculate for all fp belongs to $\mathrm{FP}_{1}$. The following figure 10 summaries $\mathrm{C}_{1}$ $\mathrm{PFP}_{1}$ patterns with their support pmf values. "_." represents not required status.

Figure 10. $C_{1}$ candidate $\mathrm{PFP}_{1}$ patterns with their support pmf values

\begin{tabular}{|l|l|l|l|l|l|l|}
\hline $\begin{array}{l}\text { SupportlPatte } \\
\text { rn }\end{array}$ & $\{\mathbf{A}\}$ & $\{\mathbf{B}\}$ & $\{\mathbf{C}\}$ & $\{\mathbf{D}\}$ & $\{\mathbf{E}\}$ & $\{\mathbf{F}\}$ \\
\hline 0 & 0 & 0 & 0 & 0.4 & 0 & 0 \\
\hline
\end{tabular}




\begin{tabular}{|l|l|l|l|l|l|l|}
1 & $\begin{array}{l}0.1 \\
2\end{array}$ & 0.09 & $\begin{array}{l}0.0 \\
6\end{array}$ & 0.6 & $\begin{array}{l}0.0 \\
9\end{array}$ & 0.036 \\
\hline 2 & $\begin{array}{l}0.4 \\
6\end{array}$ & 0.36 & $\begin{array}{l}0.2 \\
9\end{array}$ & - & $\begin{array}{l}0.3 \\
6\end{array}$ & 0.198 \\
\hline 3 & $\begin{array}{l}0.4 \\
2\end{array}$ & 0.41 & $\begin{array}{l}0.4 \\
4\end{array}$ & - & $\begin{array}{l}0.4 \\
1\end{array}$ & 0.380 \\
\hline 4 & - & 0.14 & 0.2 & - & $\begin{array}{l}0.1 \\
4\end{array}$ & 0.246 \\
\hline 5 & - & - & - & - & - & 0.140 \\
\hline
\end{tabular}

If we compare all 1-itemset support pmf values against minprob we will get PFP 1-itemset. For minprob " 0 " we will have entire Figure 10 as probabilistic frequent $\mathrm{PFP}_{1}$. So, now using all patterns in $\mathrm{PFP}_{1}$ we will continue and assign this collection to $\mathrm{L}_{1}$ and call apriori_gen on all probabilistically frequent 1-itemset patterns in $\mathrm{L}_{1}$ to generate candidate 2itemset $\mathrm{C}_{2}$. Using PDB database scan we count their support.

Figure 11. $C_{2}$ and its Support Count

\begin{tabular}{|l|l|}
\hline Pattern/2-itemset & Support in PDB \\
\hline$\{\mathrm{AB}\}$ & 2 \\
\hline$\{\mathrm{AC}\}$ & 3 \\
\hline$\{\mathrm{AE}\}$ & 2 \\
\hline$\{\mathrm{AF}\}$ & 3 \\
\hline$\{\mathrm{BC}\}$ & 3 \\
\hline$\{\mathrm{BE}\}$ & 4 \\
\hline$\{\mathrm{BF}\}$ & 4 \\
\hline$\{\mathrm{CE}\}$ & 3 \\
\hline$\{\mathrm{CF}\}$ & 4 \\
\hline$\{\mathrm{EF}\}$ & 4 \\
\hline
\end{tabular}

All pattern in Figure 11 are frequent. So, frequent 2-itemset will be as following in figure 12 .

Figure 12. $\mathrm{FP}_{2}$ and its Support Count

\begin{tabular}{|l|l|}
\hline Pattern/2-itemset & Support in PDB \\
\hline$\{\mathrm{AB}\}$ & 2 \\
\hline$\{\mathrm{AC}\}$ & 3 \\
\hline$\{\mathrm{AE}\}$ & 2 \\
\hline$\{\mathrm{AF}\}$ & 3 \\
\hline$\{\mathrm{BC}\}$ & 3 \\
\hline$\{\mathrm{BE}\}$ & 4 \\
\hline$\{\mathrm{BF}\}$ & 4 \\
\hline$\{\mathrm{CE}\}$ & 3 \\
\hline$\{\mathrm{CF}\}$ & 4 \\
\hline
\end{tabular}

$\{\mathrm{EF}\}$

4

The candidate for PFP will be as following in figure 13 .

Figure 13. $\mathrm{C}_{2}$ candidate $\mathrm{PFP}_{2}$ patterns with their support pmf values.

\begin{tabular}{|c|c|c|c|c|c|c|c|c|c|c|}
\hline $\begin{array}{l}\text { Patter } \\
\text { n> } \\
\text { Suppo } \\
\text { rtV }\end{array}$ & $\begin{array}{l}\{\mathbf{A} \\
\mathbf{B}\}\end{array}$ & $\begin{array}{l}\{\mathbf{A} \\
\mathbf{C}\}\end{array}$ & $\begin{array}{l}\{\mathbf{A E} \\
\}\end{array}$ & $\begin{array}{l}\{\mathbf{A F} \\
\}\end{array}$ & $\begin{array}{l}\{\mathbf{B} \\
\mathbf{C}\}\end{array}$ & $\begin{array}{l}\{\mathbf{B} \\
\mathbf{E}\}\end{array}$ & $\begin{array}{l}\{\mathbf{B} \\
\mathbf{F}\}\end{array}$ & $\begin{array}{l}\{\mathbf{C} \\
\mathbf{E}\}\end{array}$ & $\begin{array}{l}\{\mathbf{C} \\
\mathbf{F}\}\end{array}$ & $\begin{array}{l}\{\mathbf{E F} \\
\}\end{array}$ \\
\hline 0 & 0 & 0 & 0 & 0 & 0 & 0 & 0 & 0 & 0 & 0 \\
\hline 1 & 0.3 & $\begin{array}{l}0.1 \\
2\end{array}$ & 0.3 & 0.12 & $\begin{array}{l}0.1 \\
5\end{array}$ & $\begin{array}{l}0.0 \\
9\end{array}$ & $\begin{array}{l}0.0 \\
9\end{array}$ & $\begin{array}{l}0.1 \\
5\end{array}$ & $\begin{array}{l}0.0 \\
6\end{array}$ & 0.09 \\
\hline 2 & 0.7 & $\begin{array}{l}0.4 \\
6\end{array}$ & 0.7 & 0.46 & $\begin{array}{l}0.5 \\
0\end{array}$ & $\begin{array}{l}0.3 \\
6\end{array}$ & $\begin{array}{l}0.3 \\
6\end{array}$ & $\begin{array}{l}0.5 \\
0\end{array}$ & $\begin{array}{l}0.2 \\
9\end{array}$ & 0.36 \\
\hline 3 & - & $\begin{array}{l}0.4 \\
2\end{array}$ & - & 0.42 & $\begin{array}{l}0.3 \\
5\end{array}$ & $\begin{array}{l}0.4 \\
1\end{array}$ & $\begin{array}{l}0.4 \\
1\end{array}$ & $\begin{array}{l}0.3 \\
5\end{array}$ & $\begin{array}{l}0.4 \\
4\end{array}$ & 0.41 \\
\hline 4 & - & - & - & - & - & $\begin{array}{ll}0.1 \\
4\end{array}$ & $\begin{array}{ll}0.1 \\
4\end{array}$ & - & $\begin{array}{l}0.2 \\
1\end{array}$ & 0.14 \\
\hline 5 & - & - & - & - & - & - & - & - & - & - \\
\hline
\end{tabular}

All candidate $\mathrm{PFP}_{2}$ are probabilistic frequent hence we treat patterns in figure 13 as PFP and finally as $\mathrm{L}_{2}$. These 2-itemset probabilistically frequent patterns in $\mathrm{L}_{2}$ will be used to generate candidate 3 -itemset patterns.

Figure 14. $C_{3}$ and its Support Count

\begin{tabular}{|l|l|}
\hline Pattern/3-itemset & Support in PDB \\
\hline$\{\mathrm{ABC}\}$ & 2 \\
\hline$\{\mathrm{ABE}\}$ & 2 \\
\hline$\{\mathrm{ABF}\}$ & 2 \\
\hline$\{\mathrm{ACE}\}$ & 2 \\
\hline$\{\mathrm{ACF}\}$ & 3 \\
\hline$\{\mathrm{AEF}\}$ & 2 \\
\hline$\{\mathrm{BCE}\}$ & 3 \\
\hline$\{\mathrm{BCF}\}$ & 3 \\
\hline$\{\mathrm{BEF}\}$ & 4 \\
\hline$\{\mathrm{CEF}\}$ & 3 \\
\hline
\end{tabular}

All pattern in Figure 14 are frequent. So, frequent 3-itemset will be as following in figure 15 . 
Figure 15. $\mathbf{F P}_{3}$ and its Support Count

\begin{tabular}{|l|l|}
\hline Pattern/3-itemset & Support in PDB \\
\hline$\{A B C\}$ & 2 \\
\hline$\{A B E\}$ & 2 \\
\hline$\{A B F\}$ & 2 \\
\hline$\{A C E\}$ & 2 \\
\hline$\{A C F\}$ & 3 \\
\hline$\{A E F\}$ & 2 \\
\hline$\{B C E\}$ & 3 \\
\hline$\{B C F\}$ & 3 \\
\hline$\{B E F\}$ & 4 \\
\hline$\{C E F\}$ & 3 \\
\hline
\end{tabular}

The candidate for PFP will be as following in figure 16 .

Figure 16. $C_{3}$ candidate $\mathrm{PFP}_{3}$ patterns with their support pmf values.

\begin{tabular}{|l|l|l|l|l|l|l|l|l|l|l|}
\hline $\begin{array}{l}\text { Patte } \\
\text { rn } \\
\text { Supp } \\
\text { ort }\end{array}$ & $\begin{array}{l}\{\mathbf{A B} \\
\mathbf{C}\}\end{array}$ & $\begin{array}{l}\{\mathbf{A B} \\
\mathbf{E}\}\end{array}$ & $\begin{array}{l}\{\mathbf{A B} \\
\mathbf{F}\}\end{array}$ & $\begin{array}{l}\{\mathbf{A C} \\
\mathbf{E}\}\end{array}$ & $\begin{array}{l}\{\mathbf{A C} \\
\mathbf{F}\}\end{array}$ & $\begin{array}{l}\{\mathbf{A E} \\
\mathbf{F}\}\end{array}$ & $\begin{array}{l}\{\mathbf{B C} \\
\mathbf{E}\}\end{array}$ & $\begin{array}{l}\{\mathbf{B C} \\
\mathbf{F}\}\end{array}$ & $\begin{array}{l}\{\mathbf{B E} \\
\mathbf{F}\}\end{array}$ & $\begin{array}{l}\{\mathbf{C E} \\
\mathbf{F}\}\end{array}$ \\
\hline 0 & 0 & 0 & 0 & 0 & 0 & 0 & 0 & 0 & 0 & 0 \\
\hline 1 & $\begin{array}{l}0.26 \\
4\end{array}$ & 0.264 & $\begin{array}{l}0.26 \\
4\end{array}$ & $\begin{array}{l}0.26 \\
4\end{array}$ & $\begin{array}{l}0.26 \\
4\end{array}$ & 0.26 & 0.15 & 0.15 & 0.09 & 0.15 \\
\hline 2 & $\begin{array}{l}0.73 \\
6\end{array}$ & 0.736 & $\begin{array}{l}0.73 \\
6\end{array}$ & $\begin{array}{l}0.73 \\
6\end{array}$ & $\begin{array}{l}0.73 \\
6\end{array}$ & 0.73 & 0.50 & 0.50 & 0.36 & 0.50 \\
\hline 3 & - & - & - & - & - & - & 0.35 & 0.35 & 0.41 & 0.35 \\
\hline 4 & - & - & - & - & - & - & - & - & 0.14 & - \\
\hline 5 & - & - & - & - & - & - & - & - & - & - \\
\hline
\end{tabular}

All candidate $\mathrm{PFP}_{3}$ are probabilistic frequent hence we treat patterns in figure 16 as PFP and finally as $\mathrm{L}_{3}$. These 3-itemset probabilistically frequent patterns in $\mathrm{L}_{3}$ will be used to generate candidate 4-itemset patterns, which are as follows;

Figure 17. $C_{4}$ and its Support Count

\begin{tabular}{|l|l|}
\hline Pattern/4-itemset & Support in PDB \\
\hline$\{\mathrm{ABCE}\}$ & 2 \\
\hline$\{\mathrm{ABCF}\}$ & 2 \\
\hline$\{\mathrm{ABEF}\}$ & 2 \\
\hline$\{\mathrm{ACEF}\}$ & 2 \\
\hline$\{\mathrm{BCEF}\}$ & 3 \\
\hline
\end{tabular}

All pattern in Figure 17 are frequent. So, frequent 3-itemset will be as following in figure 18 .
Figure 18. $\mathrm{FP}_{4}$ and its Support Count

\begin{tabular}{|l|l|}
\hline Pattern/4-itemset & Support in PDB \\
\hline$\{\mathrm{ABC}\}$ & 2 \\
\hline$\{\mathrm{ABC}\}$ & 2 \\
\hline$\{\mathrm{ABEF}\}$ & 2 \\
\hline$\{\mathrm{ACEF}\}$ & 2 \\
\hline$\{\mathrm{BCEF}\}$ & 3 \\
\hline
\end{tabular}

The candidate for PFP will be as following in figure 19.

Figure 19. $\mathrm{C}_{4}$ candidate $\mathrm{PFP}_{4}$ patterns with their support pmf values.

\begin{tabular}{|l|l|l|l|l|l|}
\hline $\begin{array}{l}\text { SupportlPatte } \\
\text { rn }\end{array}$ & $\begin{array}{l}\{\text { ABCE } \\
\}\end{array}$ & $\begin{array}{l}\{\text { ABCF } \\
\}\end{array}$ & $\begin{array}{l}\text { \{ABEF } \\
\}\end{array}$ & $\begin{array}{l}\text { \{ACEF } \\
\}\end{array}$ & $\begin{array}{l}\text { \{BCEF } \\
\}\end{array}$ \\
\hline 0 & 0 & 0 & & 0 & 0 \\
\hline 1 & 0.264 & 0.264 & 0.264 & 0.264 & 0.15 \\
\hline 2 & 0.736 & 0.736 & 0.736 & 0.736 & 0.50 \\
\hline 3 & - & - & - & - & 0.35 \\
\hline 4 & - & - & - & - & - \\
\hline 5 & - & - & - & - & - \\
\hline
\end{tabular}

All candidate $\mathrm{PFP}_{4}$ are probabilistic frequent hence we treat patterns in figure 19 as PFP and finally as $\mathrm{L}_{4}$. These 4-itemset probabilistically frequent patterns in $\mathrm{L}_{4}$ will be used to generate candidate 5-itemset patterns, which are as follows;

Figure 20. $C_{5}$ and its Support Count

\begin{tabular}{|l|l|}
\hline Pattern/4-itemset & Support in PDB \\
\hline$\{\mathrm{ABCE}\}$ & 2 \\
\hline
\end{tabular}

All pattern in Figure 20 are frequent. So, frequent 3-itemset will be as following in figure 21 .

Figure 21. $\mathrm{FP}_{5}$ and its Support Count

\begin{tabular}{|l|l|}
\hline Pattern/5-itemset & Support in PDB \\
\hline$\{\mathrm{ABCEF}\}$ & 2 \\
\hline
\end{tabular}

The candidate for PFP will be as following in figure 22 .

Figure 22. $\mathrm{C}_{5}$ candidate $\mathrm{PFP}_{5}$ patterns with their support pmf values.

\begin{tabular}{|l|l|}
\hline SupportlPattern & $\{$ ABCEF $\}$ \\
\hline 0 & 0 \\
\hline 1 & 0.264 \\
\hline 2 & 0.736 \\
\hline 3 & - \\
\hline 4 & - \\
\hline 5 & - \\
\hline
\end{tabular}


All candidate $\mathrm{PFP}_{5}$ are probabilistic frequent hence we treat patterns in figure 22 as PFP and finally as $\mathrm{L}_{5}$. These 5 -itemset probabilistically frequent patterns in $\mathrm{L}_{5}$ will be used to generate candidate 6-itemset patterns. But no more new candidate 6-itemset are possible so algorithm execution stops here.

\subsection{CIPFP Algorithm}

This algorithm is an extension of probabilistic Apriori Algorithm using the concepts of key patterns and no-key patterns also known as the principle of counting inference. In the section 5.1 first we explain basic concepts of counting inference and than in section 5.2 we will describe the pseudocode for our CIPFP algorithm, in next section 5.3 we will illustrate the algorithm's functioning with the help of a toy dataset example.

\section{Basic Concepts of Counting Inference}

Key patterns and pattern Counting Inference

Definition 5.1. Let $\mathrm{P}$ be a finite set of items, $\mathrm{O}$ a finite set of objects (e.g., transaction ids) and $\mathrm{R} \subseteq \mathrm{O} \times \mathrm{P}$ a binary relation (where (o, p) $\square \mathrm{R}$ may be read as "item $\mathrm{p}$ is included in transaction o"). The triple $\mathrm{D}=(\mathrm{O}, \mathrm{P}, \mathrm{R})$ is called dataset Each subset $p$ of $P$ is called a pattern. We say that a Pattern $P$ is included in an object $\mathrm{o} \square \mathrm{O}$ if (o,p) $\square \mathrm{R}$ for all $\mathrm{p} \square \mathrm{P}$. Let $\mathrm{f}$ be the function which assign to each pattern $\mathrm{p} \subseteq \mathrm{P}$ the set of all objects which include this pattern : $\mathrm{f}(\mathrm{P})=\{\mathrm{o} \square \mathrm{O} \mid \mathrm{o}$ includes $\mathrm{P}\}$. The support of a pattern $\mathrm{P}$ is given by $\operatorname{supp}(\mathrm{P})=\operatorname{card}(\mathrm{f}$ $(\mathrm{P})) /$ card $(\mathrm{O})$. For a given threshold minsup $\square[0,1]$, a pattern $\mathrm{P}$ is called frequent pattern if $\operatorname{supp}(\mathrm{P}) \geq$ minsup.

Definition 5.2. A K-pattern $\mathrm{p}$ is a subset of $\mathrm{P}$ with $\operatorname{card}(\mathrm{P})=$ $\mathrm{K}$. A candidate K-pattern where all its proper sub-patterns are frequent.

Definition 5.3. For patterns $\mathrm{P}, \mathrm{Q} \square \mathrm{P}$, let $\mathrm{P} \theta \mathrm{Q}$ if and only if $\mathrm{f}$ $(\mathrm{P})=\mathrm{f}(\mathrm{Q})$. The set of patterns which are equivalent to a pattern $\mathrm{P}$ is given by $[\mathrm{P}]=\{\mathrm{Q} \square \mathrm{P} \mid \mathrm{P} \theta \mathrm{Q}\}$

Definition 5.4. A pattern $\mathrm{P}$ is a key pattern if $\mathrm{P} \square \min [\mathrm{P}]$. A candidate key pattern is a pattern where all its proper sub patterns are frequent key patterns.

Theorem 5A ( $\mathrm{i}$ ) if Q is a key pattern and $\mathrm{P} \square \mathrm{Q}$, then $\mathrm{P}$ is also a key pattern.

(ii) if $\mathrm{P}$ is not a key pattern and, $\mathrm{P} \square \mathrm{Q}$ then $\mathrm{Q}$ is not a key pattern either.

Theorem 5B. Let $\mathrm{P}$ be a pattern.(i) Let $\mathrm{p} \square \mathrm{P}$ then $\mathrm{P} \square[\mathrm{P}$ $\backslash\{p\}]$ if and only if $\operatorname{supp}(P)=\operatorname{supp}(P \backslash\{p\})$

(ii) $\mathrm{P}$ is a key pattern if and only if $\operatorname{supp}(\mathrm{P}) \neq \min \mathrm{p} \square \mathrm{P}$ $(\operatorname{supp}(\mathrm{P} \backslash\{\mathrm{p}\}))$

Theorem 5C. Let $\mathrm{P}$ is a non key pattern then $\operatorname{supp}(\mathrm{P})=\min \mathrm{p}$ $\mathrm{P}(\operatorname{supp}(\mathrm{P} \backslash\{\mathrm{p}\}))$

\subsection{Our CIPFP Algorithm}

Mining association rules can be decomposed into two steps: (1) finding all Frequent item sets for all transaction in probabilistic database against minsup and minprob threshold, and (2) generating Temporal association rules using the frequent item sets against given minconf threshold. The first step is the crux of the discovery of temporal association rule; in the following, we will focus on this problem. The pseudocode is given in algorithm CIPFP. The proposed algorithm is as follows,

Algorithm 1 CIPFP:
1) Ø.supp $\leftarrow 1 ; \quad \varnothing$. supp $\leftarrow$ true;

2) $\mathrm{P}_{0} \leftarrow\{\varnothing\}$;

3) $\mathrm{P}_{1} \leftarrow\{$ frequent 1-patterns $\}$;

4) forall $\mathrm{p} \in \mathrm{P}_{1}$ do begin

5) p.pred_supp $\leftarrow 1 ;$ p.key $\leftarrow($ p.supp $\neq 1)$;

6) end;

7) for $\left(\mathrm{k}=2 ; \mathrm{P}_{\mathrm{k}-1} \neq \varnothing ; \mathrm{k}++\right)$ do begin

8) $\quad \mathrm{C}_{\mathrm{k}} \leftarrow$ CIPFP-Gen $\left(\mathrm{P}_{\mathrm{k}-1}\right)$

9) if $\exists \mathrm{c} \in \mathrm{C}_{\mathrm{k}}$ |c.key then

10) forall o $\in D$ do begin

11) $\quad \mathrm{C}_{0} \leftarrow \operatorname{subset}\left(\mathrm{C}_{\mathrm{k}}, \mathrm{o}\right)$;

12) forall $\mathrm{c} \in \mathrm{C}_{0} \mid \mathrm{c} . \mathrm{key}$ do

13) c.supp++;

14) end;

15) forall $\mathrm{c} \in \mathrm{C}_{\mathrm{k}}$ do

16) if c.supp $\geq$ minsup then begin

17) if c.key and c.supp =

c.pred_supp then

18) c.key $\leftarrow$ false;

19) $\quad \mathrm{P}_{\mathrm{k}} \leftarrow \mathrm{P}_{\mathrm{k}} \mathrm{U}\{\mathrm{c}\}$;

20) end;

20a) $\quad \mathrm{FP}_{\mathrm{k}} \leftarrow \mathrm{P}_{\mathrm{k}}$

20b) forall fp $\epsilon \mathrm{FP}_{\mathrm{k}}$ do

20c) forall fp $€ \mathrm{FP}_{\mathrm{k}}$ do for each $\mathrm{k}$ from $\mathrm{k}$

$=0$ to fp.support

20d) $\quad \mathrm{W}=\mathrm{w}$ EPWS exactly size $\mathrm{k}$ times $\mathrm{k}$ number of transactions

20e) Ffp[k].prob++;

20f) end

20g) $\quad \mathrm{PFP}_{\mathrm{k}}=\left\{\mathrm{FP} \in \mathrm{FP}_{\mathrm{k}} \mid \mathrm{Ffp}[\mathrm{k}] \cdot\right.$ prob $\geq$ minprob $\}$

20h) $\quad \mathrm{P}_{\mathrm{k}}=\mathrm{PFP}_{\mathrm{k}}$

21) end;

22) return $U_{k} P_{k}$

Figure 5A CIPFP Algorithm Pseudo code

\subsection{Algorithm 2 CIPFP-Gen}

Input : $\mathrm{P}_{\mathrm{k}-1}\left(\mathrm{e}_{0}\right)$, the set of frequent $(\mathrm{K}-1)$ patterns $\mathrm{p}$ with their support p.supp and the p.key flag.

Output: $\mathrm{C}_{\mathrm{k}}\left(\mathrm{e}_{0}\right)$, the set of candidate $\mathrm{k}$ patterns $\mathrm{c}$ each with the flag c.key,the value c.pred_supp, and the support c.supp if c is not a key pattern

1) insert into $\mathrm{C}_{\mathrm{k}}\left(\mathrm{e}_{0}\right)$ select p.item $\mathrm{m}_{1}$ p.item ,......p.item $\mathrm{k-1}$, q.item $\mathrm{k-1}$

2) from $P_{k-1} p, P_{k-1} q$

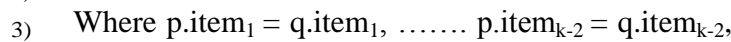
p.item $\mathrm{k}_{\mathrm{k}-1}<\mathrm{q}$. item $_{\mathrm{k}-1}$;

4)

5) for all $\mathrm{c} \square \mathrm{C}_{\mathrm{k}}\left(\mathrm{e}_{0}\right)$ do begin

6) $\quad$ c.key $\square$ true ; c.pred_supp $\square+\square$;

7) for all (k-1) subsets s of $\mathrm{c}$ do begin

8) if $\mathrm{s} \square \mathrm{P}_{\mathrm{k}-1}\left(\mathrm{e}_{0}\right)$ then

9) delete $\mathrm{c}$ from $\mathrm{C}_{\mathrm{k}}\left(\mathrm{e}_{0}\right)$

10) else begin

11) c.pred_supp $\square \min \left(c . p r e d \_s u p p, s . s u p p\right)$;

12) if not s.key then c.key $\square$ false ;

13) end;

14) end;

15) if not c.key then c.supp $\square$ c.pred_supp;

16) end;

17) return $\mathrm{C}_{\mathrm{k}}\left(\mathrm{e}_{0}\right)$

Figure 5B CIPFP -Gen Algorithm Pseudo code 
5.5 Solved Example for CIPFP Algorithms Figure 22. PDB for Pascal Transactional Temporal Dataset with Probabilistic Confidence

\begin{tabular}{|l|l|l|l|l|}
\hline TID & SetOfItems & TimeStamp & Key & $\begin{array}{l}\text { Probabilit } \\
\text { y/Confiden } \\
\text { ce }\end{array}$ \\
\hline T1 & A, B, C, F & 1 & $?$ & 0.6 \\
\hline T2 & B, C, E, F & 2 & $?$ & 0.5 \\
\hline T3 & A, B, C, E, F & 3 & $?$ & 0.7 \\
\hline T4 & B, E, F & 4 & $?$ & 0.4 \\
\hline T5 & A, B, C, E, F & 5 & $?$ & 1.0 \\
\hline
\end{tabular}

Using concepts of Possible World Semantics in earlier sections, on database presented in Figure 6 we calculated PWS, which is available in Figure 7 again displayed here in figure 23.

Figure 23. PWS for PDB for Pascal Transactional Temporal Dataset with Probabilistic Confidence

\begin{tabular}{|c|c|c|c|}
\hline $\begin{array}{l}\text { Possib } \\
\text { leWor } \\
\text { ldSem } \\
\text { antic }\end{array}$ & $\begin{array}{l}\text { TransactionIn } \\
\text { World }\end{array}$ & WorldProbalityCalculation & $\begin{array}{l}\text { World } \\
\text { Probabil } \\
\text { ity }\end{array}$ \\
\hline W1 & T5 & $\begin{array}{l}(1-0.6) *(1-0.5) *(1-0.7) *(1- \\
0.4) *(1.0)\end{array}$ & 0.036 \\
\hline W2 & T1T5 & $\begin{array}{l}\left(\begin{array}{c}0.6 \\
0.4) *(1.0)\end{array}\right) *(1-0.5) *(1-0.7) *(1- \\
\end{array}$ & 0.054 \\
\hline W3 & T2T5 & $\begin{array}{lll}(1-0.6) *( & 0.5 & )^{*}(1-0.7) *(1- \\
0.4)^{*}(1.0) & & \end{array}$ & 0.036 \\
\hline W4 & T3T5 & $\begin{array}{l}(1-0.6) *(1-0.5) *(\quad 0.7 \quad) *(1- \\
0.4) *(1.0)\end{array}$ & 0.084 \\
\hline W5 & T4T5 & $\begin{array}{l}(1-0.6) *(1-0.5) *(1-0.7) *(\quad 0.4 \\
) *(1.0)\end{array}$ & 0.024 \\
\hline W6 & T1T2T5 & $\begin{array}{l}\left.\left(\begin{array}{l}0.6 \\
0.4(\end{array}\right) * .5 \quad\right) *(1-0.7) *(1- \\
0.4)\end{array}$ & 0.054 \\
\hline W7 & T1T3T5 & 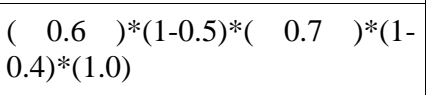 & 0.126 \\
\hline W8 & T1T4T5 & $\begin{array}{l}(0.6) *(1-0.5) *(1-0.7) *(\quad 0.4 \\
) *(1.0)\end{array}$ & 0.036 \\
\hline W9 & T2T3T5 & $\begin{array}{ll}(1-0.6) *( & 0.5) *(\quad 0.7) *(1- \\
0.4) *(1.0) & \end{array}$ & 0.084 \\
\hline W10 & T2T4T5 & $\begin{array}{l}(1-0.6) *(\quad 0.5 \quad) *(1-0.7) *(\quad 0.4 \\
) *(1.0)\end{array}$ & 0.024 \\
\hline W11 & T3T4T5 & $\begin{array}{l}(1-0.6) *(1-0.5) *(\quad 0.7 \quad) *(\quad 0.4 \\
) *(1.0)\end{array}$ & 0.056 \\
\hline
\end{tabular}

\begin{tabular}{|c|c|c|c|}
\hline W12 & T1T2T3T5 & $\begin{array}{l}\left(\begin{array}{ccc}0.6 & ) *( & 0.5\end{array}\right) *\left(\begin{array}{lll}0.7 & 0.7\end{array}\right) *(1- \\
0.4)^{*}(1.0)\end{array}$ & 0.126 \\
\hline W13 & T1T2T4T5 & $\begin{array}{l}\left.\left(\begin{array}{c}0.6 \\
) *(\end{array}\right) * .5\right) *(1-0.7) *(0.4 \\
)(1.0)\end{array}$ & 0.036 \\
\hline W14 & T1T3T4T5 & $\begin{array}{l}(0.6) *(1-0.5) *(0.7) *(0.4 \\
) *(1.0)\end{array}$ & 0.084 \\
\hline W15 & T2T3T4T5 & $\begin{array}{l}(1-0.6) *(0.5) *(0.7) *(0.4 \\
) *(1.0)\end{array}$ & 0.056 \\
\hline W16 & TIT2T3T4T5 & 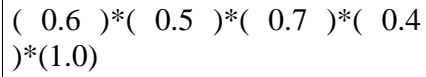 & 0.084 \\
\hline
\end{tabular}

The collection of Items for figure 7 PDB contains 6 individual items, let say $\mathrm{I}$ is the set of these items. Hence $\mathrm{I}=\{\mathrm{A}, \mathrm{B}, \mathrm{C}$, $\mathrm{D}, \mathrm{E}, \mathrm{F}$ \}, For this example minsup is $2 / 5$ that is $40 \%$. As total number of transactions in database i.e. the size of database $n$ is 5 . So, the threshold for minsup will be given by $(5 * 40)$ $/ 100$, i.e 2 . So, minsup is 2 , let minprob is 0 . We are taking minprob 0 to show that algorithm will behave exactly as apriori behave when no probabilistic transaction is considered. This is equivalent to treating all transactions having certainty or probability of 1 to occur.I

\section{Table 5.1 Notation Used In Cipfp}

\begin{tabular}{|l|l|}
\hline $\mathrm{K}$ & $\begin{array}{l}\text { Is the counter which indicates the current } \\
\text { iteration. In the Kth iteration all frequent K- } \\
\text { patterns and all key patterns among them are } \\
\text { determined. }\end{array}$ \\
\hline $\mathrm{P}_{\mathrm{k}}$ & $\begin{array}{l}\text { Contains after the Kth iteration all frequent } \mathrm{k} \\
\text { patterns P together with their support P.supp, and } \\
\text { a boolean variable P.key indicating if P is a } \\
\text { (candidate) key pattern }\end{array}$ \\
\hline $\mathrm{C}_{\mathrm{k}}$ & $\begin{array}{l}\text { Stores the candidate k patterns together with their } \\
\text { support (if Known), the Boolean variable P.key } \\
\text { and a counter P.pred_supp which stores the } \\
\text { minimum of the supports of all (k-1) sub patterns } \\
\text { of } \mathrm{P}\end{array}$ \\
\hline
\end{tabular}

All the elements which belongs to collection I will become the candidate pattern, as the individual items themselves are used as patterns we call them 1-itemset. The collection of candidate 1-itemset denoted as $C_{1}$ is as follows: $\mathrm{C}_{1}=\{\{\mathrm{A}\},\{\mathrm{B}\},\{\mathrm{C}\},\{\mathrm{D}\},\{\mathrm{E}\},\{\mathrm{F}\}\}$ for each element $\mathrm{c}$ belongs to $\mathrm{C}_{1}$ we calculate its support in database PDB Figure 6. The algorithm performs first one database pass to count the support of the 1-itemset pattern (key and non key both). The candidate pattern $\{\mathrm{D}\}$ is pruned because it is infrequent. Also $\{\mathrm{F}\}$ is marked as non key because it has the same support as empty set: Now candidate $\mathrm{C}_{1}$ and frequent set of 1itemset $P_{1}$ are see figure 24 , and figure 25 . For all itemset the PDB database scan output is as follows:

Figure 24. $C_{1}$ and its Support Count

\begin{tabular}{|l|l|}
\hline Pattern/1-itemset & Support in PDB \\
\hline$\{A\}$ & 3 \\
\hline$\{B\}$ & 4 \\
\hline
\end{tabular}




\begin{tabular}{|l|l|}
$\{\mathrm{C}\}$ & 4 \\
\hline$\{\mathrm{D}\}$ & 1 \\
\hline$\{\mathrm{E}\}$ & 4 \\
\hline$\{\mathrm{F}\}$ & 5 \\
\hline
\end{tabular}

Figure 25. P1 frequent (key \& non-key) 1-itemset with support

\begin{tabular}{|l|l|l|}
\hline Pattern/1-itemset & Support in PDB & Key \\
\hline$\{A\}$ & 3 & T \\
\hline$\{B\}$ & 4 & T \\
\hline$\{C\}$ & 4 & T \\
\hline$\{D\}$ & 1 & Pruned \\
\hline$\{E\}$ & 4 & T \\
\hline$\{F\}$ & 5 & F \\
\hline
\end{tabular}

In the figure 25 if we compare support of respective patterns we found that pattern $\{D\}$ has a support 1 which is less than the minimum support. $\sup (\{\mathrm{D}\})<$ minsup. So the set of frequent 1-itemset $\mathrm{FP}_{1}$ will contain all 1-itemset from figure 8 but not $\{\mathrm{D}\}$.This set of frequent 1-itemset $\mathrm{P}_{1}$ is stored in $\mathrm{FP}_{1}$ is as is given in figure 26 .

Figure $26 \mathbf{F P}_{1}$

\begin{tabular}{|l|l|l|}
\hline Pattern/1-itemset & Support in PDB & Key \\
\hline$\{A\}$ & $3 / 5$ & T \\
\hline$\{B\}$ & $4 / 5$ & T \\
\hline$\{C\}$ & $4 / 5$ & T \\
\hline$\{E\}$ & $4 / 5$ & T \\
\hline$\{F\}$ & 1 & F \\
\hline
\end{tabular}

Now we use patterns in $\mathrm{FP}_{1}$ one by one to calculate their existential probability. For this we first scan PDB and found the TIDs in which pattern is present. For combination of length equal to in range for support from 0 to c.support we start scan of PWS rows and sum up the provabilities of Wi in which exactly the number of transaction present, here the number of transaction which occurred in Wi is determined for every support from 0 to c.support. For example we want to determine existential probability of frequent 1 -itemset $\{A\}$ to determine probabilistic frequent pattern $\{\mathrm{A}\}$ is or not. First, we scan PDB and found that T1, T3, T5 contains $\{A\}$. $\{\mathrm{A}\}$.support is 3 . So for transaction combination length of 0 , 1,2 , and 3 , for example example length 1 means any Wi that contain exactly one time any of the $\{\mathrm{A}\}$.Ti, i.e., Wi exactly contain either one of $\mathrm{T} 1$, or $\mathrm{T} 2$, or $\mathrm{T} 2$ but not $\mathrm{T} 1 \mathrm{~T} 2$, $\mathrm{T} 1 \mathrm{~T} 3$ or $\mathrm{T} 2 \mathrm{~T} 3$ or T1T2T3 together, all their probabilities will be summed up. For $\{\mathrm{A}\}$.support is 1 , PWS is W1W3W5W10 with probability $0.036,0.036,0.024,0.024$ summed up to
0.12 , this 0.12 is existential probability of pattern $\{\mathrm{A}\}$ when its support is 1 . Like wise we calculate for all $\mathrm{fp}$ belongs to $\mathrm{FP}_{1}$. The following figure 10 summaries $\mathrm{C}_{1} \mathrm{PFP}_{1}$ patterns with their support pmf values. "-" represents not required status.

Figure 27. $C_{1}$ candidate PFP 1-itemset patterns with their support pmf values

\begin{tabular}{|c|c|c|c|c|c|c|}
\hline $\begin{array}{l}\text { Pattern> } \\
\text { SupportV }\end{array}$ & $\{\mathbf{A}\}$ & $\{B\}$ & $\{C\}$ & $\{D\}$ & $\{\mathbf{E}\}$ & $\{F\}$ \\
\hline 0 & 0 & 0 & 0 & 0.4 & 0 & 0 \\
\hline 1 & 0.12 & 0.09 & 0.06 & 0.6 & 0.09 & 0.036 \\
\hline 2 & 0.46 & 0.36 & 0.29 & - & 0.36 & 0.198 \\
\hline 3 & 0.42 & 0.41 & 0.44 & - & 0.41 & 0.380 \\
\hline 4 & - & 0.14 & 0.21 & - & 0.14 & 0.246 \\
\hline 5 & - & - & - & - & - & 0.140 \\
\hline
\end{tabular}

If we compare all 1-itemset frequent probabilistic candidate support pmf values against minprob we will get PFP 1itemset. For minprob "0" we will have entire Figure 10 as probabilistic frequent $\mathrm{PFP}_{1}$. So, now using all patterns in $\mathrm{PFP}_{1}$ we will continue and assign this collection to update P1 and now we call CIPFP_Gen on all probabilistically frequent 1 itemset patterns in $\mathrm{P}_{1}$ to generate candidate 2-itemset $\mathrm{C}_{2}$. Using PDB we count their support. At the next iteration, all candidate 2-itemset patterns are created and stored in $\mathrm{C}_{2}$, key and non-key elements are categorized and probabilistic support pmf is evaluated. at the same time the support of pattern containing infrequent pattern $\{\mathrm{F}\}$ as sub-pattern is computed. Then a database pass is performed to determine the support of the remaining six candidate patterns. Hence $\mathrm{P}_{2}$ achieved: Now $\mathrm{C}_{2}$ is see figure 28 ,

Figure 28. $\mathrm{C}_{2}$ and its Support Count From this we have Now $P_{2}$ is see Figure 29.

\begin{tabular}{|l|l|l|l|}
\hline $\begin{array}{l}\text { Pattern/2- } \\
\text { itemset }\end{array}$ & pred_supp & Key & supp \\
\hline$\{\mathrm{AB}\}$ & $3 / 5$ & $T$ & $?$ \\
\hline$\{\mathrm{AC}\}$ & $3 / 5$ & $\mathrm{~T}$ & $?$ \\
\hline$\{\mathrm{AE}\}$ & $4 / 5$ & $T$ & $?$ \\
\hline$\{\mathrm{AF}\}$ & $3 / 5$ & $\mathrm{~F}$ & $3 / 5$ \\
\hline$\{\mathrm{BC}\}$ & $4 / 5$ & $\mathrm{~T}$ & $?$ \\
\hline$\{\mathrm{BE}\}$ & $4 / 5$ & $\mathrm{~T}$ & $?$ \\
\hline$\{\mathrm{BF}\}$ & $4 / 5$ & $\mathrm{~F}$ & $?$ \\
\hline$\{\mathrm{CE}\}$ & $4 / 5$ & $T$ & $?$ \\
\hline$\{\mathrm{CF}\}$ & $4 / 5$ & $\mathrm{~F}$ & $?$ \\
\hline$\{\mathrm{EF}\}$ & $4 / 5$ & $\mathrm{~F}$ & $?$ \\
\hline
\end{tabular}


Figure $29 \mathrm{P}_{2}$ frequent (key \& non-key) 2-itemset with support

\begin{tabular}{|l|l|l|}
\hline $\begin{array}{l}\text { Pattern/2- } \\
\text { itemset }\end{array}$ & supp & Key \\
\hline$\{\mathrm{AB}\}$ & $2 / 5$ & $\mathrm{~T}$ \\
\hline$\{\mathrm{AC}\}$ & $3 / 5$ & $\mathrm{~F}$ \\
\hline$\{\mathrm{AE}\}$ & $2 / 5$ & $\mathrm{~T}$ \\
\hline$\{\mathrm{AF}\}$ & $3 / 5$ & $\mathrm{~F}$ \\
\hline$\{\mathrm{BC}\}$ & $3 / 5$ & $\mathrm{~T}$ \\
\hline$\{\mathrm{BE}\}$ & $4 / 5$ & $\mathrm{~F}$ \\
\hline$\{\mathrm{BF}\}$ & $4 / 5$ & $\mathrm{~F}$ \\
\hline$\{\mathrm{CE}\}$ & $3 / 5$ & $\mathrm{~T}$ \\
\hline$\{\mathrm{CF}\}$ & $4 / 5$ & $\mathrm{~F}$ \\
\hline$\{\mathrm{EF}\}$ & $4 / 5$ & $\mathrm{~F}$ \\
\hline
\end{tabular}

All pattern in Figure 29 are frequent. So, frequent 2-itemset will be as following in figure 30 .

Figure 30. FP $_{2}$ and its Support Count

\begin{tabular}{|l|l|}
\hline Pattern/2-itemset & Support in PDB \\
\hline$\{A B\}$ & 2 \\
\hline$\{A C\}$ & 3 \\
\hline$\{A E\}$ & 2 \\
\hline$\{A F\}$ & 3 \\
\hline$\{B C\}$ & 3 \\
\hline$\{B E\}$ & 4 \\
\hline$\{B F\}$ & 4 \\
\hline$\{C E\}$ & 3 \\
\hline$\{C F\}$ & 4 \\
\hline$\{E F\}$ & 4 \\
\hline
\end{tabular}

The candidate for $\mathrm{PFP}_{2}$ will be as following in figure 31 .

Figure 31. $\mathrm{C}_{2}$ candidate $\mathbf{P F P}_{2}$ patterns with their support pmf values.

\begin{tabular}{|c|c|c|c|c|c|c|c|c|c|c|}
\hline $\begin{array}{l}\text { Patter } \\
\text { n> } \\
\text { Suppor } \\
\text { tV }\end{array}$ & $\begin{array}{l}\{\mathbf{A} \\
\mathbf{B}\}\end{array}$ & $\begin{array}{l}\{\mathrm{A} \\
\mathrm{C}\}\end{array}$ & $\begin{array}{l}\{\mathbf{A E} \\
\}\end{array}$ & $\begin{array}{l}\{\mathbf{A} \\
\mathbf{F}\}\end{array}$ & $\begin{array}{l}\{\mathrm{BC} \\
\}\end{array}$ & $\begin{array}{l}\{\mathbf{B} \\
\mathbf{E}\}\end{array}$ & $\begin{array}{l}\{\mathbf{B} \\
\mathbf{F}\}\end{array}$ & $\begin{array}{l}\{\mathrm{CE} \\
\}\end{array}$ & $\begin{array}{l}\{\mathbf{C F} \\
\}\end{array}$ & $\left\{\begin{array}{l}\{\mathbf{E F} \\
\}\end{array}\right.$ \\
\hline 0 & 0 & 0 & 0 & 0 & 0 & 0 & 0 & 0 & 0 & 0 \\
\hline 1 & 0.3 & $\begin{array}{l}0.1 \\
2\end{array}$ & 0.3 & $\begin{array}{l}0.1 \\
2\end{array}$ & 0.15 & $\begin{array}{l}0.0 \\
9\end{array}$ & $\begin{array}{l}0.0 \\
9\end{array}$ & 0.15 & 0.06 & 0.09 \\
\hline 2 & 0.7 & $\begin{array}{l}0.4 \\
6\end{array}$ & 0.7 & $\begin{array}{l}0.4 \\
6\end{array}$ & 0.50 & $\begin{array}{l}0.3 \\
6\end{array}$ & $\begin{array}{l}0.3 \\
6\end{array}$ & 0.50 & 0.29 & 0.36 \\
\hline 3 & - & $\begin{array}{l}0.4 \\
2\end{array}$ & - & $\begin{array}{l}0.4 \\
2\end{array}$ & 0.35 & \begin{tabular}{|l}
0.4 \\
1
\end{tabular} & $\begin{array}{l}0.4 \\
1\end{array}$ & 0.35 & 0.44 & 0.41 \\
\hline 4 & - & - & - & - & - & \begin{tabular}{|l}
0.1 \\
4
\end{tabular} & $\begin{array}{l}0.1 \\
4\end{array}$ & - & 0.21 & 0.14 \\
\hline 5 & - & - & - & - & - & - & - & - & - & - \\
\hline
\end{tabular}

All candidate- $\mathrm{PFP}_{2}$ are probabilistic frequent hence we treat all patterns in figure 31 as $\mathrm{PFP}_{2}$ and finally update as $\mathrm{P}_{2}$.
These 2-itemset probabilistically frequent patterns in $\mathrm{P}_{2}$ will be used to generate candidate 3-itemset patterns. Repeating again we have Now $\mathrm{C}_{3}$.

Figure 32. $C_{3}$ and its Support Count,

\begin{tabular}{|l|l|l|l|}
\hline $\begin{array}{l}\text { Pattern/3- } \\
\text { itemset }\end{array}$ & pred_supp & Key & supp \\
\hline$\{\mathrm{ABC}\}$ & $2 / 5$ & F & $2 / 5$ \\
\hline$\{\mathrm{ABE}\}$ & $2 / 5$ & F & $2 / 5$ \\
\hline$\{\mathrm{ABF}\}$ & $2 / 5$ & F & $2 / 5$ \\
\hline$\{\mathrm{ACE}\}$ & $2 / 5$ & F & $2 / 5$ \\
\hline$\{\mathrm{ACF}\}$ & $3 / 5$ & $\mathrm{~F}$ & $3 / 5$ \\
\hline$\{\mathrm{AEF}\}$ & $2 / 5$ & $\mathrm{~F}$ & $2 / 5$ \\
\hline$\{\mathrm{BCE}\}$ & $3 / 5$ & $\mathrm{~F}$ & $3 / 5$ \\
\hline$\{\mathrm{BCF}\}$ & $3 / 5$ & $\mathrm{~F}$ & $3 / 5$ \\
\hline$\{\mathrm{BEF}\}$ & $4 / 5$ & F & $4 / 5$ \\
\hline$\{\mathrm{CEF}\}$ & $3 / 5$ & F & $3 / 5$ \\
\hline
\end{tabular}

All pattern in Figure 32 are frequent. So, frequent 3-itemset will be as following in figure 33

Figure 33. FP $_{3}$ and its Support Count

\begin{tabular}{|l|l|}
\hline Pattern/3-itemset & Support in PDB \\
\hline$\{A B C\}$ & 2 \\
\hline$\{A B E\}$ & 2 \\
\hline$\{A B F\}$ & 2 \\
\hline$\{A C E\}$ & 3 \\
\hline$\{A C F\}$ & 2 \\
\hline$\{A E F\}$ & 3 \\
\hline$\{B C E\}$ & 3 \\
\hline$\{B C F\}$ & 4 \\
\hline$\{B E F\}$ & 3 \\
\hline$\{C E F\}$ & \\
\hline
\end{tabular}

The candidate for PFP will be as following in figure 33. As all patterns are non-key in candidyate- $\mathrm{PFP}_{3}$. From now onwards no database scan is required at all to count support. We compute support of higher level pattern using their already determined support of sub patterns. Minimum of support of sub-patterns is used as the support of higher level pattern. 
Figure 34. $C_{3}$ candidate $\mathrm{PFP}_{3}$ patterns with their support pmf values.

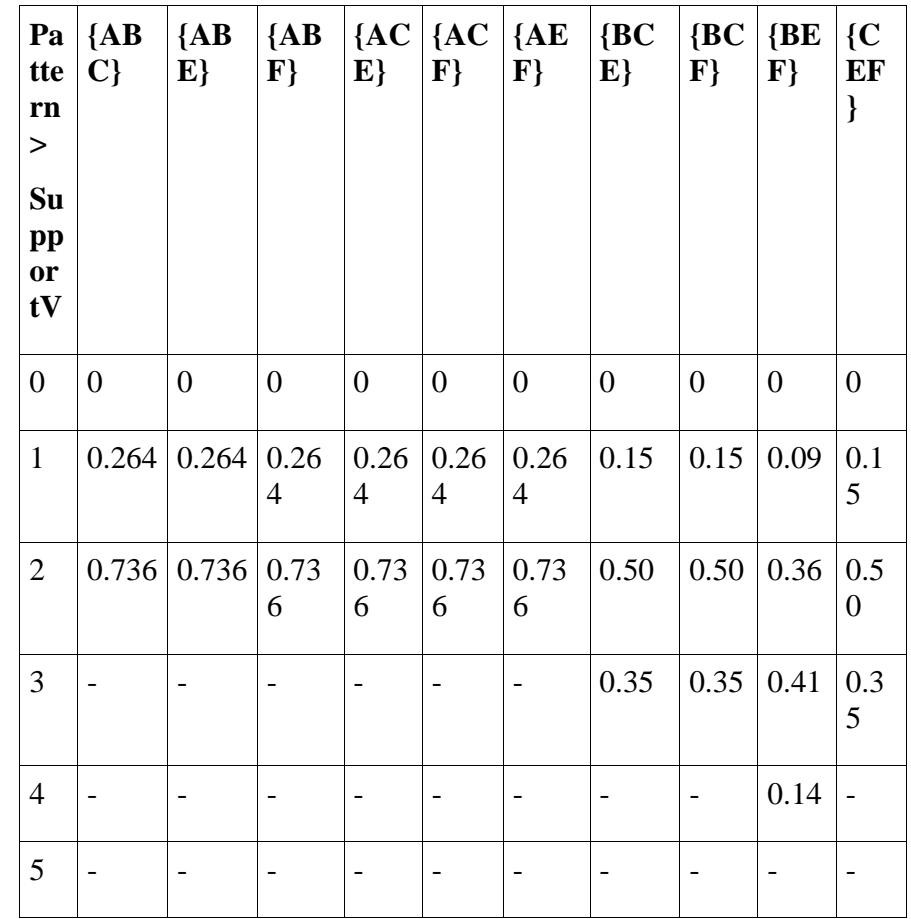

As all candidate- $\mathrm{PFP}_{3}$ are probabilistic frequent hence we treat patterns in figure 34 as $\mathrm{PFP}_{3}$ and finally update $\mathrm{P}_{3}$ by $\mathrm{PFP}_{3}$. These 3-itemset probabilistically frequent patterns in $\mathrm{P}_{3}$ will be used to generate candidate 4-itemset patterns, which are as follows see figure 35 . So now in $4^{\text {th }}$ and $5^{\text {th }}$ iteration no database scan is required as we have no key candidate to calculate support in database.

Figure 35. $C_{4}$ candidate (key \& non-key) 4-itemset with support

\begin{tabular}{|l|l|l|l|}
\hline $\begin{array}{l}\text { Pattern/4- } \\
\text { itemset }\end{array}$ & pred_supp & Key & supp \\
\hline$\{\mathrm{ABCE}\}$ & $2 / 5$ & $\mathrm{~F}$ & $2 / 5$ \\
\hline$\{\mathrm{ABCF}\}$ & $2 / 5$ & $\mathrm{~F}$ & $2 / 5$ \\
\hline$\{\mathrm{ABEF}\}$ & $2 / 5$ & $\mathrm{~F}$ & $2 / 5$ \\
\hline$\{\mathrm{ACEF}\}$ & $2 / 5$ & $\mathrm{~F}$ & $2 / 5$ \\
\hline$\{\mathrm{BCEF}\}$ & $3 / 5$ & $\mathrm{~F}$ & $3 / 5$ \\
\hline
\end{tabular}

All pattern in Figure 35 are frequent. So, frequent 3-itemset will be as following in figure 36 .

Figure 36. $\mathrm{FP}_{4}$ frequent (key \& non-key) 4-itemset with support

\begin{tabular}{|l|l|l|}
\hline Pattern/4-itemset & supp & Key \\
\hline$\{\mathrm{ABCE}\}$ & $2 / 5$ & F \\
\hline$\{\mathrm{ABCF}\}$ & $2 / 5$ & F \\
\hline$\{\mathrm{ABEF}\}$ & $2 / 5$ & F \\
\hline$\{\mathrm{ACEF}\}$ & $2 / 5$ & F \\
\hline$\{\mathrm{BCEF}\}$ & $3 / 5$ & F \\
\hline
\end{tabular}

The candidate for PFP will be as following in figure 37 .
Figure 37. $\mathrm{C}_{4}$ candidate $\mathrm{PFP}_{4}$ patterns with their support pmf values

\begin{tabular}{|l|l|l|l|l|l|}
\hline $\begin{array}{l}\text { Pattern } \\
\text { SupportV }\end{array}$ & $\{\mathrm{ABCE}\}$ & $\{\mathrm{ABCF}\}$ & $\begin{array}{l}\{\mathrm{ABEF} \\
\}\end{array}$ & $\begin{array}{l}\{\mathrm{ACEF} \\
\}\end{array}$ & $\{\mathrm{BCEF}\}$ \\
\hline 0 & 0 & 0 & & 0 & 0 \\
\hline 1 & 0.264 & 0.264 & 0.264 & 0.264 & 0.15 \\
\hline 2 & 0.736 & 0.736 & 0.736 & 0.736 & 0.50 \\
\hline 3 & - & - & - & - & 0.35 \\
\hline 4 & - & - & - & - & - \\
\hline 5 & - & - & - & - & - \\
\hline
\end{tabular}

All candidate $\mathrm{PFP}_{4}$ are probabilistic frequent hence we treat patterns in figure 37 as probabilistically frequent patterns and finally update $\mathrm{P}_{4}$ by $\mathrm{PFP}_{4}$. These 4-itemset probabilistically frequent patterns in $\mathrm{P}_{4}$ will be used to generate candidate 5itemset patterns, which are as follows;

Figure 38. $C_{5}$ candidate (key \& non-key) 5-itemset with support

\begin{tabular}{|l|l|l|l|}
\hline $\begin{array}{l}\text { Pattern/5- } \\
\text { itemset }\end{array}$ & pred_supp & Key & supp \\
\hline$\{\mathrm{ABCEF}\}$ & $2 / 5$ & $\mathrm{~F}$ & $2 / 5$ \\
\hline
\end{tabular}

All pattern in Figure 38 are frequent. So, frequent 5-itemset will be as following in figure 39 .

Figure 39. FP $_{5}$ frequent (key \& non-key) 5-itemset with support

\begin{tabular}{|l|l|l|}
\hline $\begin{array}{l}\text { Pattern/5- } \\
\text { itemset }\end{array}$ & Key & supp \\
\hline$\{\mathrm{ABCEF}\}$ & $\mathrm{F}$ & $2 / 5$ \\
\hline
\end{tabular}

The candidate for PFP will be as following in figure 40 .

Figure 40. $C_{5}$ candidate $\mathbf{P F P}_{5}$ patterns with their support pmf values

\begin{tabular}{|l|l|}
\hline Support Pattern & $\{$ ABCEF $\}$ \\
\hline 0 & 0 \\
\hline 1 & 0.264 \\
\hline 2 & 0.736 \\
\hline 3 & - \\
\hline 4 & - \\
\hline 5 & - \\
\hline
\end{tabular}

All candidate- $\mathrm{PFP}_{5}$ are having all kth support smf probability greater than minprob hence we treat all of the patterns in figure 40 as probabilistically frequent patterns set $\mathrm{PFP}_{5}$ and finally update $\mathrm{P}_{5}$ by $\mathrm{PFP}_{5}$. These 5-itemset probabilistically frequent patterns in $\mathrm{P}_{5}$ will be used to generate candidate 6itemset patterns. But no more new candidate 6-iteset are possible so algorithm execution stops here.

\section{CONCLUSION}

Real world application requires mining of patterns occurrences of which are random or uncertain in nature. This uncertain is introduced in the system because of many parameters which may be endogenous or exogenous. This uncertainty is introduced many time because of uncertainty in 
competency of operator involved in transactional process. Uncertainty may be introduced due to limitation of measurement machines, instruments, and/or procedure. So, to encompass this truth of process we studied and suggested a mining procedure that can be used to answer queries which or otherwise only possible to answer on certain data using data mining techniques. To best of our knowledge the steps we carried out are not discuss to this much extent in any paper. Algorithm finally covered. 32 probabilistic frequent patterns were generated when we took the probability of transactions equal to one, i.e. minimum threshold is considered as 0. Many variations in implementation of the algorithm are possible. Also how to select suitable minprob threshold is, depending on this algorithm can be modified. In future we will implement the algorithm with possible alternate implementation as well as mechanism to support selection of minimum support threshold, and selection of minimum probability threshold. The number of DB scan in case of Apriori and probabilistic Apriori are 5, and pattern to be cross checked where 32. But in case of CIPFP algorithm only two database scan are required and only 12 patterns are required to be checked for support in database. Hence CIPFP needs two database passes in which the algorithm counted the support of $6+6=12$ patterns. Apriori and Probabilistic Apriori would have needed five database passes for counting supports of $6+6+10+10+5+1=32$ patterns for the same dataset. In future we are going to develop and test concept on more databases from variety of domains.

\section{REFERENCES}

[1] R. Agrawal, C. Faloutsos, and A. Swami. Efcient similarity search in sequence databases. In Proc. of the Fourth International Conference on Foundations of Data Organization and Algorithms, Chicago, October 1993.

[2] R. Agrawal, S. Ghosh, T. Imielinski, B. Iyer, and A. Swami. An interval classi er for database mining applications. In Proc. of the VLDB Conference, pages 560\{573, Vancouver, British Columbia, Canada, 1992.

[3] R. Agrawal, T. Imielinski, and A. Swami. Database mining: A performance perspective. IEEE Transactions on Knowledge and Data En- gineering, 5(6):914\{925, December 1993. Special Issue on Learning and Discovery in Knowledge- Based Databases.

[4] R. Agrawal, T. Imielinski, and A. Swami. Mining association rules between sets of items in large databases. In Proc. of the ACM SIGMOD Con- ference on Management of Data, Washington, D.C., May 1993.

[5] R. Agrawal and R. Srikant. Fast algorithms for mining association rules in large databases. Re- search Report RJ 9839, IBM Almaden Research Center, San Jose, California, June 1994.

[6] D. S. Associates. The new direct marketing. Business One Irwin, Illinois, 1990.

[7] R. Brachman et al. Integrated support for data archeology. In AAAI-93 Workshop on Knowledge Discovery in Databases, July 1993.

[8] L. Breiman, J. H. Friedman, R. A. Olshen, and C. J. Stone. Classi cation and Regression Trees. Wadsworth, Belmont, 1984
[9] P. Cheeseman et al. Autoclass: A bayesian classi cation system. In 5th Int'1 Conf. on Machine Learning. Morgan Kaufman, June 1988.

[10] D. H. Fisher. Knowledge acquisition via incremental conceptual clustering. Machine Learning, 2(2), 1987.

[11] J. Han, Y. Cai, and N. Cercone. Knowledge discovery in databases: An attribute oriented approach. In Proc. of the VLDB Conference, pages 547\{559, Vancouver, British Columbia, Canada, 1992.

[12] M. Holsheimer and A. Siebes. Data mining: The search for knowledge in databases. Technical Report CSR9406, CWI, Netherlands, 1994

[13] M. Houtsma and A. Swami. Set-oriented mining of association rules. Research Report RJ 9567, IBM Almaden Research Center, San Jose, Cali- fornia, October 1993.

[14] R. Krishnamurthy and T. Imielinski. Practi- tioner problems in need of database research: Re- search directions in knowledge discovery. SIG- MOD RECORD, 20(3):76\{78, September 1991.

[15] P. Langley, H. Simon, G. Bradshaw, andJ. Zytkow. Scienti c Discovery: Computational Explorations of the Creative Process. MIT Press, 1987.

[16] H. Mannila and K.-J. Raiha. Dependency inference. In Proc. of the VLDB Conference, pages $155\{158$, Brighton, England, 1987.

[17] H. Mannila, H. Toivonen, and A. I. Verkamo. E cient algorithms for discovering association rules. In KDD-94: AAAI Workshop on Knowl- edge Discovery in Databases, July 1994.

[18] S. Muggleton and C. Feng. E cient induction of logic programs. In S. Muggleton, editor, Inductive Logic Programming. Academic Press, 1992.

[19] J. Pearl. Probabilistic reasoning in intelligent systems: Networks of plausible inference, 1992.

[20] G. Piatestsky-Shapiro. Discovery, analy- sis, and presentation of strong rules. In G. Piatestsky-Shapiro, editor, Knowledge Dis- covery in Databases. AAAI/MIT Press, 1991.

[21] G. Piatestsky-Shapiro, editor. Knowledge Dis- covery in Databases. AAAI/MIT Press, 1991.

[22] J. R. Quinlan. C4.5: Programs for Machine Learning. Morgan Kaufman, 1993.

[23] Rakesh Agrawal, Ramakrishnan Srikant: "Fast Algorithms for Mining Association Rules" Proceedings of the 20th VLDB Conference Santiago, Chile, 1994

[24] Liwen Sun, Reynold Cheng, David W. Cheung, Jiefeng Cheng, "Mining Uncertain Data with Probabilistic Guarantees", KDD'10, July 25-28, 2010, Washington, DC, USA. Copyright 2010 ACM 978-1-4503-0055$1 / 10 / 07$

[25] A. Deshpande et al. Model-driven data acquisition in sensor networks. In VLDB, 2004.

[26] C. Aggarwal, Y. Li, J. Wang, and J. Wang. Frequent pattern mining with uncertain data. In KDD, 2009. 
[27] C. Aggarwal and P. Yu. A survey of uncertain data algorithms and applications. IEEE Transactions on Knowledge and Data Engineering, 21(5), 2009.

[28] R. Agrawal and R. Srikant. Fast algorithms for mining association rules in large databases. Technical report, RJ 9839, IBM, 1994.

[29] R. Bayardo, Jr. Efficiently mining long patterns from databases. In SIGMOD, 1998.

[30] D. Burdick, M. Calimlim, J. Flannick, J. Gehrke, and T. Yiu. MAFIA: A maximal frequent itemset algorithm. IEEE Transactions on Knowledge and Data Engineering, 17, 2005.

[31] H. Cheng, P. Yu, and J. Han. Approximate frequent itemset mining in the presence of random noise. Soft Computing for Knowledge Discovery and Data Mining, 2008 .

[32] R. Cheng, D. Kalashnikov, and S. Prabhakar. Evaluating probabilistic queries over imprecise data. In SIGMOD, 2003.

[33] C. K. Chui, B. Kao, and E. Hung. Mining frequent itemsets from uncertain data. In PAKDD, 2007.

[34] G. Cormode and M. Garofalakis. Sketching probabilistic data streams. In SIGMOD, 2007.

[35] N. Dalvi and D. Suciu. Efficient query evaluation on probabilistic databases. In VLDB, 2004

[36] M. Garofalakis and A. Kumar. Wavelet synopses for general error metrics. ACM Transactions on Database Systems, 30(4), 2005.

[37] K. Gouda and M. J. Zaki. GenMax: An efficient algorithm for mining maximal frequent itemsets. Data Mining and Knowledge Discovery, 11(3), 2005.

[38] R. Hogg, A. Craig, and J. Mckean. Introduction to Mathematical Statistics (6th ed.). Prentice Hall, 2004.

[39] J. Huang et al. MayBMS: A Probabilistic Database Management System. In SIGMOD, 2009.

[40] N. Khoussainova, M. Balazinska, and D. Suciu.Towards correcting input data errors probabilistically using integrity constraints. In MobiDE, 2006.

[41] D. Knuth. The art of computer programming, vol. 3.Addison Wesley, 1998.

[42] H. Kriegel and M. Pfeifle. Density-based clustering of uncertain data. In KDD, 2005.

[43] C. Kuok, A. Fu, and M. Wong. Mining fuzzy association rules in databases. SIGMOD Record, 1998.
[44] A. Lu, Y. Ke, J. Cheng, and W. Ng. Mining vague association rules. In DASFAA, 2007.

[45] M. Mutsuzaki et al. Trio-one: Layering uncertainty and lineage on a conventional dbms. In CIDR, 2007.

[46] M. Yiu et al. Efficient evaluation of probabilistic advanced spatial queries on existentially uncertain data. IEEE Transactions on Knowledge and Data Engineering, 21(9), 2009

[47] R. Motwani and P. Raghavan. Randomized algorithms. Cambridge University Press, New York, NY, USA, 1995.

[48] A. Oppenheim, R. Schafer, and J. Buck. Discrete-time signal processing (2nd ed.). Prentice Hall, 1999.

[49] P. Sistla et al. Querying the uncertain position of moving objects. In Temporal Databases: Research and Practice. Springer Verlag, 1998.

[50] J. Ren, S. Lee, X. Chen, B. Kao, R. Cheng, and D. Cheung. Na"1ve Bayes Classification of Uncertain Data. In ICDM, 2009.

[51] T. Bernecker et al. Probabilistic frequent itemset mining in uncertain databases. In KDD, 2009.

[52] T. Jayram et al. Avatar information extraction system. IEEE Data Engineering Bulletin, 29(1), 2006.

[53] P. Tan, M. Steinbach, and V. Kumar. Introduction to Data Mining. Pearson Education, 2006.

[54] C. Yang and W. Najm. Examining driver behavior using data gathered from red light photo enforcement cameras. Journal of Safety Research, 38(3), 2007.

[55] Q. Zhang, F. Li, and K. Yi. Finding frequent items in probabilistic data. In SIGMOD, 2008.

\section{AUTHORS PROFILE}

Niket Bhargava, born in state is MP in the India, on July 6, 1978. He graduated from the Oriental Institute of Science And Technology, Bhopal, and after qualifying in GATE, completed his Master in Technology from the RGTU- the technical university of MP, India. His employment experience included the 10 years as Teacher in Top Most Ranking Institutions of state of MP,and Industry experience of BI product development. Presently, he is pursuing his $\mathrm{PhD}$ in CSE with focus on business applications of data mining, data science, big data techniques for Business Intelligence.

Dr. Manoj Shukla, is working as professor in BCE, Mandideep. He is having more than 15 years of experience in teaching and hold positions like principal, and others in various institutes in Madhya Pradesh, India 